\title{
Effect of Remixing \& Intermittent Curing Sequences on Strength of Pavement Quality Concrete
}

\author{
Vishal B Shinde, Rakesh Kumar
}

\begin{abstract}
The paper is concerned with determining the effect of remixing of two different grades of concrete proving blend ratio, time lag \& intermittent curing sequence. Two mix designs (M40 \& M50) selected and provide blend ratio (0 to infinity) at 0,30,60,90 \& 120min time lag. In this research, the problem related to improper casting \& curing sequence, which ultimate formation of the cold joint due to old and fresh concrete. Curing method applied with four sequences Air0 Water28, Air28 Water0, Air7 Water21, and Air21 Water7.Concrete samples were made and tested for their compressive strength, flexural strength, elastic modulus, and shrinkage properties in the laboratory. As compared to the strength of freshly prepared concretes to the hard in situ concrete tends to show a reduction in its various strengths due to improper curing \& formation of cold joints. This reduction is further possible to be minimized to a certain extent on blending some quantity of a relatively fresh mix to the existing quantity of the hard in situ concrete.
\end{abstract}

Key word: Cold joint, intermittent curing, blend ratio, Time lag.

\section{INTRODUCTION}

Hydration process provides strength of concrete [20 \& 23]. The hydration process is closely related to concrete microstructure development, proper curing measures are necessary to maintain satisfactory moisture and temperature condition [4 \& 11]. Normally 28 days continuous curing on the laboratory gives a satisfactory result to achieve the required strength of concrete. But in practice that complete 28 days water curing is not possible due to various reasons [2]. The different types of curing (Air, water, steam etc.) and its effect on strength on concrete for various grades of concrete. Adequate curing is necessary with the proper method of its application for a newly placed concrete to achieve the enviable qualities and accepted durability of the hardened concrete [5 \& 15]. Curing in the early ages of concrete is more important for concrete structures. Though the exact lab curing practice is not possible on the field, up to a certain extent the suggested methods can be applied in due consideration of site conditions[6]. Curing may be applied in several ways and the most appropriate means of curing may be dictated by the site or the construction method[3]. The curing condition i.e. standard curing, natural curing, water

Revised Manuscript Received on April 25, 2020.

* Correspondence Author

Vishal B Shinde*, Research scholar, TEP, Civil Engineering Dept. at Sardar Vallabhbhai Patel National Institute of Technology, Surat, Gujarat, India, Email: shindevishal747@gmail.com

Rakesh Kumar, Professor, TEP, Civil Engineering Dept. at Sardar Vallabhbhai Patel National Institute of Technology, Surat, Gujarat, Email: krakesh1999@gmail.com

(c) The Authors. Published by Blue Eyes Intelligence Engineering and Sciences Publication (BEIESP). This is an open access article under the CC BY-NC-ND license (http://creativecommons.org/licenses/by-nc-nd/4.0/) curing, and sealed curing, on the capillary absorption of normal concrete has an important influence on the compressive strength, ultrasonic pulse velocity, and porosity[10]. To improve the intermittent curing adverse effect selfing and crossing methodology was adapted. In this methodology reuse the preset concrete by adding fresh concrete to form a remix by considering their time lags, blend ratio and various curing sequences [1 \& 2]. Sometime due to delay in the placing of concrete or some transportation problems or accidents happened on site, delay placing in formwork, the mix is often declared to be rejected. Cement is a costly ingredient in concrete so its wastage is to be avoided and try to reuse of wastage concrete, yes it is useful to investigate up to what magnitude the old concrete be made durable on producing it efficiently rather than fully rejected. In the selfing and crossing method, we used the improvement of preset concrete mixed by adding higher grade or same grade of fresh concrete considering time lag. In selfing and crossing method blending action of two grades of concrete mixes, one is relatively old concrete (Co) than the fresh concrete of same grade mix or higher grade mix concrete (Cf). The ratio of (Co) / (Cf) known as blend ratio (r). [1]. The strength of various curing period of alternate dry and wet curing of concrete three times a day is much more sufficient and it does not reduce the compressive strength after 28 days in comparison to full-time curing [7].

\section{A.Effect of intermittent curing in the strength of PQC}

Appropriate curing is most important in mass concreting to produce high-quality pavement concrete. In mass concreting mostly concentrate on the hydration process of cement, for that controlled water-cement ratio as well as maintain proper moisture \& temperature condition. [8]. Internally cured concrete (ICC) used recently in pavement work they found ICC pavement achieved 28 days flexural strength within 7 days also ICC reduced the concrete early strength \& escalate the ultimate strength.[9\&17]. The early edge properties of concrete pavement (Like Temperature, moisture, hydration rate, etc.) are important in his durability life point of view, supplementary cementitious materials (SCMs) are being increasingly used in the concrete pavement due to its economic and environmental benefits, and improvements of concrete properties, such as workability, impermeability, ultimate strength and durability [11 \& 12]. There are also some factors which cause the delaying process is that improper method of handling, work schedule, lack of site organization and the breakdown of equipment. To overcome such problems, the process of remixing concrete, if necessary, with the addition of just the required quantity of water is known as „retemperingee of concrete1. Sometimes, a small quantity of extra cement is also added while retempering.[13\&14].

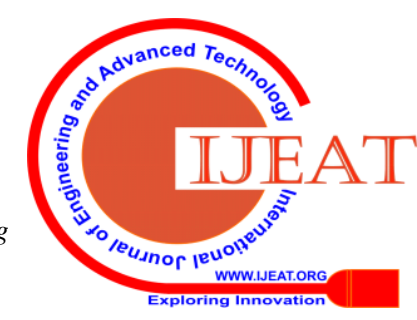




\section{Experimental Programme}

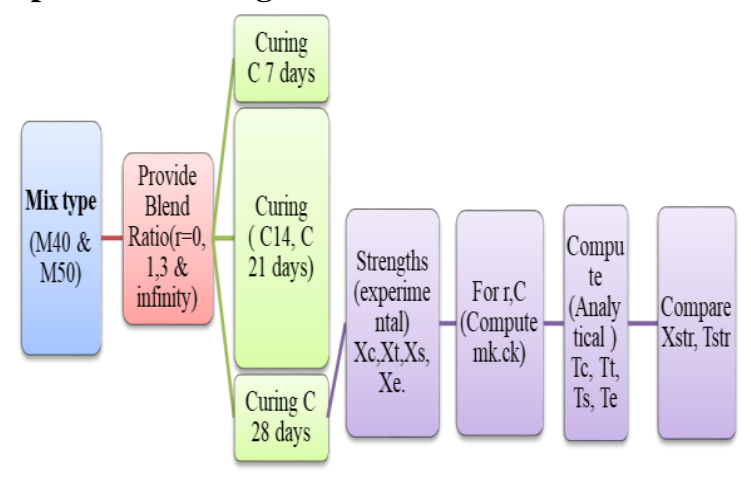

Fig 1. Flow Chart

$\mathrm{Xc}_{\mathrm{c}}=$ Compressive strength (Experimental)

$\mathrm{Xt}=$ Tensile strength $($ Experimental $)$

$\mathrm{Xs}=$ Shear strength (Experimental)

$\mathrm{Xe}=$ Modulus of elasticity (Experimental)

C7,C14,C21 \& C28 Curing are regular \& irregular curing.

For verification of Selfing and Crossing concepts and to build up a database consisting of characteristic strength parameters, it is necessary to study the high strength concrete mixes of M40 \& M50.

To study the effects of intermittent curing on strength of rigid pavement concrete slab cube specimen by considering parameters like water-cement ratio, mix type, different time lag and to a particular target strength either by Selfing it with a freshly prepared mix of known strength at any instant of time lag \& against any improper curing sequence type. For compressive strength, cubes of dimension 150 x 150 x150 mm were cast and tested as per IS 516: 1959. \& foe tensile strength Cylinder of dimension $150 \mathrm{~mm}$ diameter \& 300mm height were cast \& tested.

Concrete cubes \& cylinder cast by providing different blend ratio, time lag and cured under different curing conditions. Considered the curing conditions are, A00W28, $\mathrm{A}_{07} \mathrm{~W}_{21}, \mathrm{~W}_{14} \mathrm{~A}_{14}, \mathrm{~A}_{21} \mathrm{~W}_{07}, \mathrm{~A}_{28} \mathrm{~W}_{00}$, for various blend ratio viz., $\mathrm{r}=\infty, 3.00,1.00$ and 0 as well as time lag $\mathrm{t}$ (minute) 0.00. $30.00,60.00,90.00,120.00$ and $150.00 \mathrm{~min}$ is used for the test.All the specimens were demolded the next day and put to curing for 'the specified periods in water as per the various planned, improper curing sequences as mentioned above. The specimens to be cured for the specified partial water curing were taken out of water and stored outside in air for the designated period. The 28-day water cured specimens were, however, tested soon after taking out from water after or on swapping the water. Completely air-cured and partially water-cured specimens were, however, tested in dry condition. The cube and cylinder specimens were tested on 100 ton and 200 -ton- capacity machine after suitable calibration in each case.

The cubes and cylinder specimens were tested for compressive strength and modulus of elasticity as per the method specified in IS: 516-1959 (Methods of test for strength of concrete and the cylinder specimens were tested for split tensile strength in accordance with the prescribed procedure in IS:5816-1970 (Methods of test for split tensile strength of concrete cylinders). The average value of the compressive strength and modulus of elasticity have been calculated by testing 3 cubes and 3 cylinder specimens respectively.

\section{MATERIALS AND TEST SPECIMENS}

Ordinary Portland cement conforming to Indian Standard .IS: 269 -1976 has been used for all the mixes. River-sand and crushed granite coarse aggregate in Metal have been used. For normal concrete mixes, the grading of fine and coarse aggregates conformed to the grading requirements specified in IS: 383-1910 (Specification for coarse and fine aggregates from natural sources _for concrete). For high strength concrete, a type grading curve has been used. The type grading curve was arrived at by carrying out a series of pilot tests in which the proportions of coarse to fine aggregates. Metal 1 and Metal 2, A/C ratios and W/C ratios were varied. The results obtained from the Pilot tests conducted are given in Table IV \& Table V. The specific gravities and bulk densities of the fine and coarse aggregates used were determined as per 2386 (Part III) - 1963 (Methods of Test for Aggregate for Concrete) and found to be 2.62 and 2.63 and $1615 \mathrm{~kg} / \mathrm{m} 3$ and $1587 \mathrm{~kg} / \mathrm{m} 3$ respectively. The aggregates used were in a saturated surface dry condition. The control test specimens comprised of $150 \mathrm{~mm}$ standard cubes and 150 $\mathrm{mm} 300 \mathrm{~mm}$ cylinders for the determination of the compressive strength, split tensile strength And modulus of elasticity of all the concrete types cured under the following insufficient curing ( cumulative curing ) sequences. A0W28, A7W21, A21W7, A28W00 W28A00.

\section{A.Cement}

Cement is a binding material used in construction. It has property setting and hardening when mixed with water to attain strength cement is always used in the form of either grout or mortar or concrete, so we use cement of OPC 53 grade for concreting.

The initial and final setting time of cement have been observed, through standard Vicat needle apparatus as per IS:4031- 1968 ( Method of physical tests tor hydraulic cement) and are as follows:

Initial setting time of cement $=40$ minute

Final setting time of cement $=280$ minute

The compressive strength values of the cement used observed at 3, 7 and 28 days-of complete water curing.

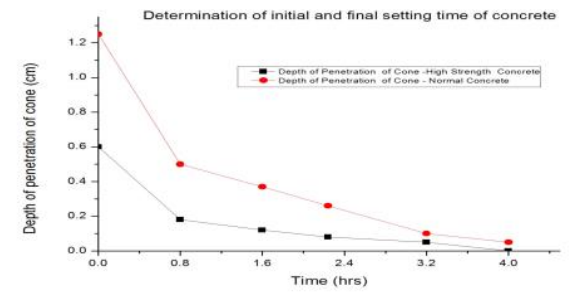

Fig 2 Determination of IST \& FST.

\section{B. Aggregate}

Aggregates are the major ingredient of the concrete which occupies $70 \%$ to $80 \%$ of the volume of concrete. Aggregate provides strength to concrete and act as filler material to give a homogeneous mass of concrete along with cement paste.

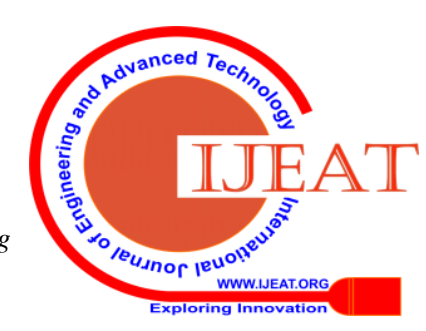


Table- I: Properties of Aggregate

\begin{tabular}{|c|c|c|}
\hline Sr. No. & Properties & Normal Aggregate \\
\hline 1 & Specific Gravity & $2.78(\mathrm{CA})$ \& 2.63(FA) \\
\hline 2 & Bulk Density & $1.487 \mathrm{~kg} /$ lit (CA) \\
\hline 3 & Impact Value & $17.64 \%(\mathrm{CA})$ \\
\hline 4 & Fineness Modulus & $6.97(\mathrm{CA})$ \\
\hline 5 & Water Absorption & $1.2 \%(\mathrm{CA})$ \\
\hline 6 & Moisture Content & $37 \%$ \\
\hline
\end{tabular}

Table - II: Mix Proportion of concrete

\begin{tabular}{|c|c|c|c|c|}
\hline \multicolumn{5}{|c|}{ Trial Mix Ratio } \\
\hline Grade & W/C & Cement & $\begin{array}{c}\text { Fine } \\
\text { Aggregate }\end{array}$ & $\begin{array}{c}\text { Coarse } \\
\text { Aggregate }\end{array}$ \\
\hline M 40 & 0.40 & 1 & 1.82 & 3.09 \\
\hline M 50 & 0.35 & 1 & 1.43 & 2.42 \\
\hline
\end{tabular}

Table - III: Specimens details

\begin{tabular}{|l|l|l|l|l|}
\hline $\begin{array}{l}\text { Speci } \\
\text { mens }\end{array}$ & $\begin{array}{l}\text { Conve-nti } \\
\text { onal } \\
\text { BL0 }\end{array}$ & $\begin{array}{l}\text { Selfi } \\
\text { ng } \\
\text { BL1 }\end{array}$ & $\begin{array}{l}\text { Selfing } \\
\mathrm{BL}_{3}\end{array}$ & $\begin{array}{l}\text { Selfing } \\
\text { BLInfinity }\end{array}$ \\
\hline Cube & 15 & 75 & 75 & 75 \\
\hline $\begin{array}{l}\text { Cylli } \\
\text { nder }\end{array}$ & 15 & 75 & 75 & 75 \\
\hline
\end{tabular}

Table - IV: Pilot test results of compressive strength of concrete mix for different ratios of $\mathrm{CA}$ : FA and for $\mathrm{A} / \mathrm{C}$ $=3.0, \mathrm{~W} / \mathrm{C}=\mathbf{0 . 3 0}$

\begin{tabular}{|l|c|l|c|l|l|l|}
\hline \multirow{2}{*}{$\begin{array}{c}\text { Time } \\
\text { lag for } \\
\text { basic } \\
\text { mix }\end{array}$} & \multicolumn{5}{|c|}{ Compressive strength in $\mathrm{MP}^{*} 10$ and Density in $\mathrm{kg} / \mathrm{m}^{3}$} \\
\cline { 2 - 7 } & $70: 30$ & Density & $75: 25$ & Density & $80: 20$ & Density \\
\hline $\mathrm{t}_{0}$ & 53.90 & 2653 & 56.80 & 2648 & 54.90 & 2660 \\
\hline $\mathrm{t}_{\mathrm{i}}$ & 57.70 & 2697 & 60.10 & 2699 & 58.00 & 2695 \\
\hline $\mathrm{t}_{\mathrm{f}}$ & 52.00 & 2589 & 49.55 & 2580 & 52.25 & 2593 \\
\hline
\end{tabular}

Table V: Pilot test results of compressive strength of concrete mix for different ratios of $\mathrm{CA}$ : FA and for $\mathrm{A} / \mathrm{C}$ $=2.5, \mathrm{~W} / \mathrm{C}=0.28$

\begin{tabular}{|c|c|l|l|l|l|l|}
\hline \multirow{2}{*}{$\begin{array}{c}\text { Time } \\
\text { lag } \\
\text { for } \\
\text { basic } \\
\text { mix }\end{array}$} & \multicolumn{6}{|c|}{ Compressive strength in $\mathrm{MPa}^{*} 10$ and Density in $\mathrm{kg} / \mathrm{m}^{3}$} \\
\hline $\mathrm{t}_{\mathrm{o}}$ & 58.50 & 2650 & 61.00 & 2650 & 59.60 & 2645 \\
\hline $\mathrm{t}_{\mathrm{i}}$ & 64.80 & 2710 & 63.00 & 2695 & 61.70 & 2688 \\
\hline $\mathrm{t}_{\mathrm{f}}$ & 53.00 & 2575 & 54.70 & 2590 & 53.90 & 2598 \\
\hline
\end{tabular}

\section{RESULTS AND DISCUSSIONS}

The individual types considered within the scope of the present investigation are concreting of normal as well as higher grades each of these mix types again cured under various cumulative improper curing sequences. The computation for the prediction of the strengths analytically following various steps of calculation using proper analytical. procedure for any two appropriate combinations of the preset mix cases in blending by using repetitively the selfing equation is really an impossible task if intended to be carried out manually.

Table VI: Details of work for Cubes (Curing Sequences)

\begin{tabular}{|c|c|c|c|}
\hline Sr.no & Blend ratio ( $r$ ) & Time lag (t) Minute & Curing Sequences \\
\hline 1 & 0 & 0 & $\mathrm{~A}_{00} \mathrm{~W}_{28}, \mathrm{~A}_{07} \mathrm{~W}_{21}, \mathrm{~A}_{14} \mathrm{~W}_{14}, \mathrm{~A}_{21} \mathrm{~W}_{07}, \mathrm{~A}_{28} \mathrm{~W}_{00}$ \\
\hline \multirow{5}{*}{1} & \multirow{5}{*}{1} & 30 & $\mathrm{~A}_{00} \mathrm{~W}_{28}, \mathrm{~A}_{07} \mathrm{~W}_{21}, \mathrm{~A}_{14} \mathrm{~W}_{14}, \mathrm{~A}_{21} \mathrm{~W}_{07}, \mathrm{~A}_{28} \mathrm{~W}_{00}$ \\
\hline & & 60 & $\mathrm{~A}_{00} \mathrm{~W}_{28}, \mathrm{~A}_{07} \mathrm{~W}_{21}, \mathrm{~A}_{14} \mathrm{~W}_{14}, \mathrm{~A}_{21} \mathrm{~W}_{07}, \mathrm{~A}_{28} \mathrm{~W}_{00}$ \\
\hline & & 90 & $\mathrm{~A}_{00} \mathrm{~W}_{28}, \mathrm{~A}_{07} \mathrm{~W}_{21}, \mathrm{~A}_{14} \mathrm{~W}_{14}, \mathrm{~A}_{21} \mathrm{~W}_{07}, \mathrm{~A}_{28} \mathrm{~W}_{00}$ \\
\hline & & 120 & $\mathrm{~A}_{00} \mathrm{~W}_{28}, \mathrm{~A}_{07} \mathrm{~W}_{21}, \mathrm{~A}_{14} \mathrm{~W}_{14}, \mathrm{~A}_{21} \mathrm{~W}_{07}, \mathrm{~A}_{28} \mathrm{~W}_{00}$ \\
\hline & & 150 & $\mathrm{~A}_{00} \mathrm{~W}_{28}, \mathrm{~A}_{07} \mathrm{~W}_{21}, \mathrm{~A}_{14} \mathrm{~W}_{14}, \mathrm{~A}_{21} \mathrm{~W}_{07}, \mathrm{~A}_{28} \mathrm{~W}_{00}$ \\
\hline \multirow{5}{*}{2} & \multirow{5}{*}{3} & 30 & $\mathrm{~A}_{00} \mathrm{~W}_{28}, \mathrm{~A}_{07} \mathrm{~W}_{21}, \mathrm{~A}_{14} \mathrm{~W}_{14}, \mathrm{~A}_{21} \mathrm{~W}_{07}, \mathrm{~A}_{28} \mathrm{~W}_{00}$ \\
\hline & & 60 & $\mathrm{~A}_{00} \mathrm{~W}_{28}, \mathrm{~A}_{07} \mathrm{~W}_{21}, \mathrm{~A}_{14} \mathrm{~W}_{14}, \mathrm{~A}_{21} \mathrm{~W}_{07}, \mathrm{~A}_{28} \mathrm{~W}_{00}$ \\
\hline & & 90 & $\mathrm{~A}_{00} \mathrm{~W}_{28}, \mathrm{~A}_{07} \mathrm{~W}_{21}, \mathrm{~A}_{14} \mathrm{~W}_{14}, \mathrm{~A}_{21} \mathrm{~W}_{07}, \mathrm{~A}_{28} \mathrm{~W}_{00}$ \\
\hline & & 120 & $\mathrm{~A}_{00} \mathrm{~W}_{28}, \mathrm{~A}_{07} \mathrm{~W}_{21}, \mathrm{~A}_{14} \mathrm{~W}_{14}, \mathrm{~A}_{21} \mathrm{~W}_{07}, \mathrm{~A}_{28} \mathrm{~W}_{00}$ \\
\hline & & 150 & $\mathrm{~A}_{00} \mathrm{~W}_{28}, \mathrm{~A}_{07} \mathrm{~W}_{21}, \mathrm{~A}_{14} \mathrm{~W}_{14}, \mathrm{~A}_{21} \mathrm{~W}_{07}, \mathrm{~A}_{28} \mathrm{~W}_{00}$ \\
\hline \multirow{5}{*}{4} & \multirow{5}{*}{ infinity } & 30 & $\mathrm{~A}_{00} \mathrm{~W}_{28}, \mathrm{~A}_{07} \mathrm{~W}_{21}, \mathrm{~A}_{14} \mathrm{~W}_{14}, \mathrm{~A}_{21} \mathrm{~W}_{07}, \mathrm{~A}_{28} \mathrm{~W}_{00}$ \\
\hline & & 60 & $\mathrm{~A}_{00} \mathrm{~W}_{28}, \mathrm{~A}_{07} \mathrm{~W}_{21}, \mathrm{~A}_{14} \mathrm{~W}_{14}, \mathrm{~A}_{21} \mathrm{~W}_{07}, \mathrm{~A}_{28} \mathrm{~W}_{00}$ \\
\hline & & 90 & $\mathrm{~A}_{00} \mathrm{~W}_{28}, \mathrm{~A}_{07} \mathrm{~W}_{21}, \mathrm{~A}_{14} \mathrm{~W}_{14}, \mathrm{~A}_{21} \mathrm{~W}_{07}, \mathrm{~A}_{28} \mathrm{~W}_{00}$ \\
\hline & & 120 & $\mathrm{~A}_{00} \mathrm{~W}_{28}, \mathrm{~A}_{07} \mathrm{~W}_{21}, \mathrm{~A}_{14} \mathrm{~W}_{14}, \mathrm{~A}_{21} \mathrm{~W}_{07}, \mathrm{~A}_{28} \mathrm{~W}_{00}$ \\
\hline & & 150 & $\mathrm{~A}_{00} \mathrm{~W}_{28}, \mathrm{~A}_{07} \mathrm{~W}_{21}, \mathrm{~A}_{14} \mathrm{~W}_{14}, \mathrm{~A}_{21} \mathrm{~W}_{07}, \mathrm{~A}_{28} \mathrm{~W}_{00}$ \\
\hline
\end{tabular}

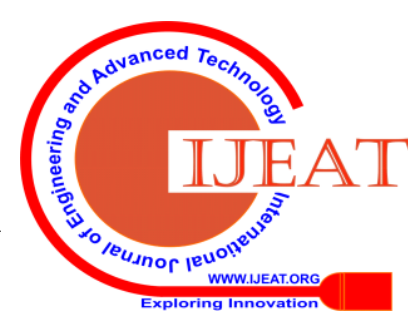


Table VII: Comparison between the analytical and experimental values of Selfed strength of Mix M40 for different time lag parameters (Type of Curing A28W00).

\begin{tabular}{|c|c|c|c|c|c|c|c|c|c|c|c|}
\hline \multirow{2}{*}{$\begin{array}{c}\text { Type } \\
\text { of } \\
\text { Curing }\end{array}$} & \multirow{2}{*}{$\begin{array}{c}\text { Blend } \\
\text { Ratio } \\
\text { (r) }\end{array}$} & \multirow{2}{*}{$\begin{array}{c}\text { Time } \\
\text { Lag } \\
\text { (Min.) } \\
\text { t }\end{array}$} & \multicolumn{3}{|c|}{$\begin{array}{l}\text { Compressive strength } \\
\left(\mathbf{N} / \mathbf{m m}^{2}\right)\end{array}$} & \multicolumn{3}{|c|}{ Tensile strength(N/mm²) } & \multicolumn{3}{|c|}{$\begin{array}{l}\text { Modulus of Elasticity in } \\
\text { Gpa) }\end{array}$} \\
\hline & & & $\mathrm{XC}(\mathrm{t})$ & $A C(t)$ & $\begin{array}{c}\% \\
\text { Error }\end{array}$ & $X T(t)$ & $\mathbf{A T}(\mathbf{t})$ & $\begin{array}{c}\% \\
\text { Error }\end{array}$ & $\mathrm{XE}(\mathrm{t})$ & $\mathrm{AE}(\mathrm{t})$ & $\begin{array}{c}\% \\
\text { Error }\end{array}$ \\
\hline \multirow{24}{*}{$\mathbf{A}_{28} \mathbf{W}_{00}$} & 0 & 0 & 40.59 & 40.59 & 0.00 & 3.76 & 3.76 & 0.00 & 27.18 & 27.18 & 0.00 \\
\hline & 0 & 30 & 41.39 & 41.29 & -0.24 & 3.81 & 3.80 & -0.08 & 27.35 & 27.33 & -0.07 \\
\hline & 0 & 60 & 41.29 & 41.18 & -0.27 & 3.80 & 3.79 & -0.11 & 27.33 & 27.29 & -0.15 \\
\hline & 0 & 90 & 40.89 & 40.81 & -0.19 & 3.78 & 3.78 & -0.13 & 27.24 & 27.23 & -0.04 \\
\hline & 0 & 120 & 40.59 & 40.55 & -0.09 & 3.76 & 3.76 & 0.00 & 27.18 & 27.17 & -0.04 \\
\hline & 0 & 150 & 40.19 & 40.29 & 0.25 & 3.75 & 3.75 & 0.00 & 27.09 & 27.11 & 0.07 \\
\hline & 1 & 0 & 40.59 & 40.59 & 0.00 & 3.76 & 3.76 & 0.00 & 27.18 & 27.18 & 0.00 \\
\hline & 1 & 30 & 41.79 & 40.81 & -2.40 & 3.82 & 3.82 & -0.10 & 27.44 & 27.42 & -0.07 \\
\hline & 1 & 60 & 41.19 & 41.28 & 0.22 & 3.80 & 3.80 & 0.03 & 27.33 & 27.33 & 0.00 \\
\hline & 1 & 90 & 40.59 & 40.63 & 0.10 & 3.78 & 3.75 & -0.80 & 27.22 & 27.21 & -0.04 \\
\hline & 1 & 120 & 40.19 & 40.20 & 0.03 & 3.75 & 3.75 & 0.00 & 27.09 & 27.09 & 0.00 \\
\hline & 1 & 150 & 30.69 & 30.69 & 0.00 & 3.72 & 3.72 & 0.00 & 26.97 & 26.97 & 0.00 \\
\hline & 3 & 0 & 40.59 & 40.59 & 0.00 & 3.76 & 3.76 & 0.00 & 27.18 & 27.18 & 0.00 \\
\hline & 3 & 30 & 42.29 & 42.13 & -0.38 & 3.85 & 3.82 & -0.63 & 27.55 & 27.51 & -0.15 \\
\hline & 3 & 60 & 41.19 & 41.44 & 0.60 & 3.80 & 3.80 & 0.16 & 27.42 & 27.37 & -0.18 \\
\hline & 3 & 90 & 40.39 & 40.63 & 0.59 & 3.75 & 3.77 & 0.53 & 27.35 & 27.18 & -0.63 \\
\hline & 3 & 120 & 39.69 & 39.79 & 0.25 & 3.73 & 3.73 & 0.19 & 26.99 & 27.01 & 0.07 \\
\hline & 3 & 150 & 38.99 & 39.09 & 0.27 & 3.70 & 3.70 & 0.00 & 26.81 & 26.83 & 0.07 \\
\hline & $\infty$ & 0 & 39.87 & 39.87 & 0.00 & 3.76 & 3.76 & 0.00 & 27.18 & 27.18 & 0.00 \\
\hline & $\infty$ & 30 & 42.29 & 42.18 & -0.26 & 3.84 & 3.84 & 0.00 & 27.55 & 27.53 & -0.07 \\
\hline & $\infty$ & 60 & 39.99 & 40.05 & 0.16 & 3.75 & 3.77 & 0.64 & 27.27 & 27.20 & -0.26 \\
\hline & $\infty$ & 90 & 38.39 & 39.22 & 2.12 & 3.68 & 3.70 & 0.59 & 26.89 & 26.88 & -0.04 \\
\hline & $\infty$ & 120 & 37.39 & 38.69 & 3.36 & 3.62 & 3.61 & -0.30 & 26.58 & 26.55 & -0.11 \\
\hline & $\infty$ & 150 & 36.49 & 36.49 & 0.00 & 3.57 & 3.57 & 0.00 & 26.22 & 26.22 & 0.00 \\
\hline
\end{tabular}

Table VIII: Comparison between the analytical and experimental values of Selfed strength of Mix M40 for different time lag parameters (Type of Curing A21W07).

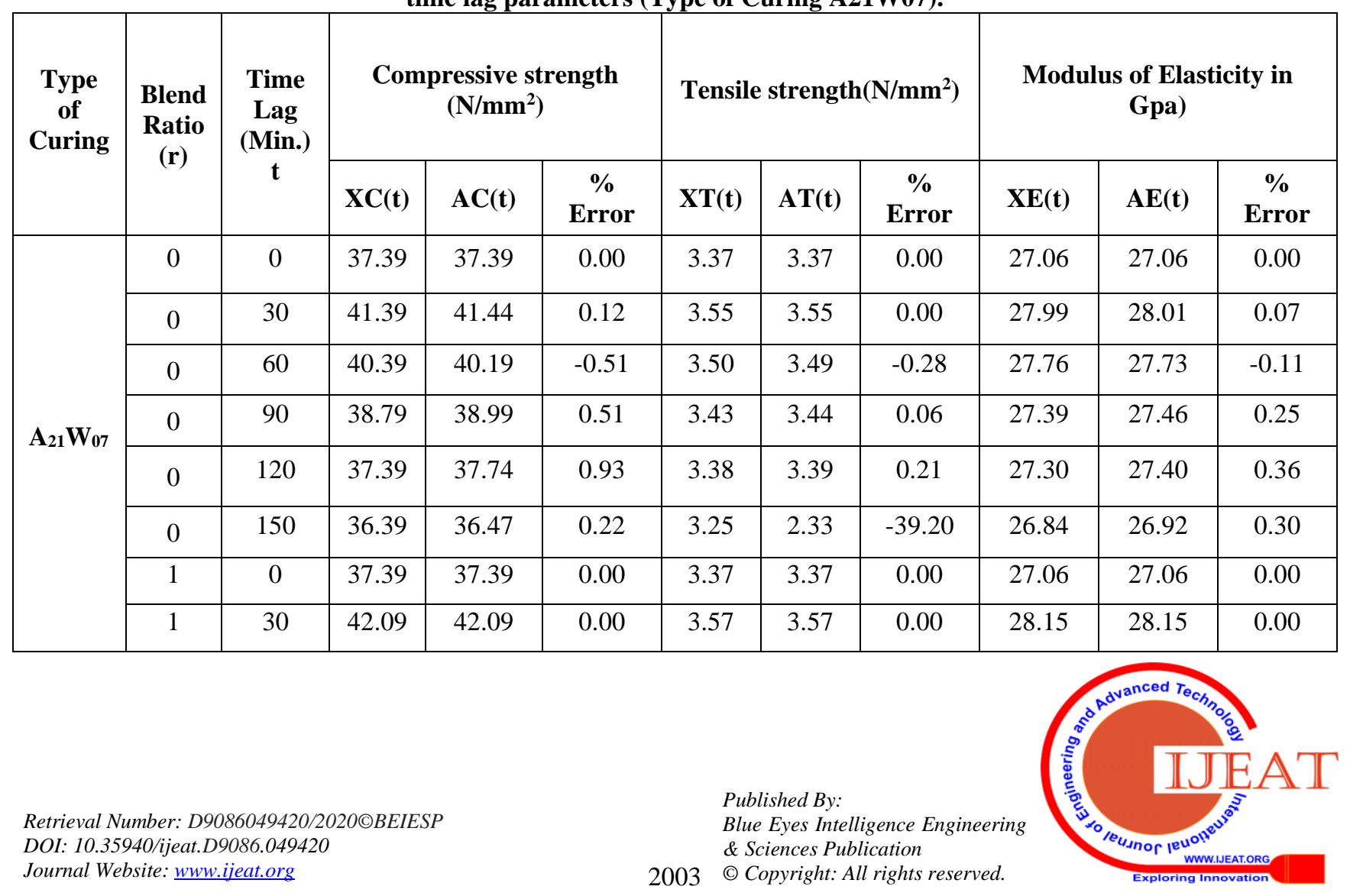




\begin{tabular}{|c|c|c|c|c|c|c|c|c|c|c|c|}
\hline 1 & 60 & 39.89 & 39.89 & 0.00 & 3.48 & 3.52 & 1.25 & 27.56 & 27.70 & 0.51 \\
\hline 1 & 90 & 37.89 & 38.15 & 0.69 & 3.40 & 3.45 & 1.62 & 27.18 & 27.27 & 0.33 \\
\hline 1 & 120 & 36.09 & 36.45 & 0.98 & 3.32 & 3.35 & 1.16 & 26.74 & 26.85 & 0.41 \\
\hline 1 & 150 & 34.89 & 34.89 & 0.00 & 3.24 & 3.24 & 0.00 & 26.44 & 26.44 & 0.00 \\
\hline 3 & 0 & 37.39 & 37.39 & 0.00 & 3.37 & 3.37 & 0.00 & 27.06 & 27.06 & 0.00 \\
\hline 3 & 30 & 42.69 & 42.73 & 0.09 & 3.59 & 3.59 & 0.00 & 28.28 & 28.29 & 0.04 \\
\hline 3 & 60 & 38.79 & 39.78 & 2.49 & 3.44 & 3.47 & 0.84 & 27.60 & 27.67 & 0.25 \\
\hline 3 & 90 & 36.19 & 37.21 & 2.74 & 3.32 & 3.35 & 1.07 & 26.76 & 27.07 & 1.15 \\
\hline 3 & 120 & 34.29 & 34.95 & 1.90 & 3.22 & 3.50 & 7.86 & 26.29 & 26.50 & 0.79 \\
\hline 3 & 150 & 32.89 & 32.89 & 0.00 & 3.15 & 3.15 & 0.03 & 25.93 & 25.95 & 0.08 \\
\hline$\infty$ & 0 & 37.39 & 37.39 & 0.00 & 3.37 & 3.37 & 0.00 & 27.06 & 27.06 & 0.00 \\
\hline$\infty$ & 30 & 43.09 & 43.09 & 0.00 & 3.62 & 3.62 & 0.00 & 28.37 & 28.37 & 0.00 \\
\hline$\infty$ & 60 & 36.09 & 37.59 & 3.99 & 3.09 & 3.21 & 3.80 & 28.09 & 28.16 & 0.25 \\
\hline$\infty$ & 90 & 31.99 & 33.29 & 3.91 & 2.79 & 2.88 & 3.33 & 27.83 & 27.95 & 0.43 \\
\hline$\infty$ & 120 & 29.19 & 29.95 & 2.54 & 2.56 & 2.67 & 4.16 & 27.61 & 27.83 & 0.79 \\
\hline$\infty$ & 150 & 27.19 & 27.19 & 0.00 & 2.40 & 2.40 & 0.00 & 27.55 & 27.55 & 0.00 \\
\hline
\end{tabular}

Table IX: Comparison between the analytical and experimental values of Selfed strength of Mix M40 for different time lag parameters (Type of Curing $\mathrm{A}_{14} \mathrm{~W}_{14}$ ).

\begin{tabular}{|c|c|c|c|c|c|c|c|c|c|c|c|}
\hline \multirow{2}{*}{$\begin{array}{l}\text { Type of } \\
\text { Curing }\end{array}$} & \multirow{2}{*}{$\begin{array}{l}\text { Blend } \\
\text { Ratio } \\
\text { (r) }\end{array}$} & \multirow{2}{*}{$\begin{array}{l}\text { Time } \\
\text { Lag } \\
\text { (Min.) } \\
\text { t }\end{array}$} & \multicolumn{3}{|c|}{$\begin{array}{l}\text { Compressive strength } \\
\text { (N/mm2) }\end{array}$} & \multicolumn{3}{|c|}{ Tensile strength(N/mm2) } & \multicolumn{3}{|c|}{ Modulus of Elasticity in Gpa) } \\
\hline & & & $\mathrm{XC}(\mathrm{t})$ & AC(t) & $\begin{array}{l}\% \\
\text { Error }\end{array}$ & $\mathrm{XT}(\mathrm{t})$ & $\mathbf{A T}(\mathbf{t})$ & $\begin{array}{c}\% \\
\text { Error }\end{array}$ & $\mathrm{XE}(\mathrm{t})$ & $\mathbf{A E}(\mathrm{t})$ & $\begin{array}{c}\% \\
\text { Error }\end{array}$ \\
\hline \multirow{15}{*}{$A_{14} W_{14}$} & 0 & 0 & 39.23 & 39.23 & 0.00 & 3.39 & 3.39 & 0.00 & 26.86 & 26.86 & 0.00 \\
\hline & 0 & 30 & 42.03 & 41.97 & -0.14 & 3.51 & 3.51 & 0.00 & 27.49 & 27.48 & -0.04 \\
\hline & 0 & 60 & 40.63 & 40.93 & 0.73 & 3.46 & 3.47 & 0.17 & 27.36 & 27.26 & -0.37 \\
\hline & 0 & 90 & 39.43 & 39.93 & 1.25 & 3.41 & 3.47 & 1.81 & 27.14 & 27.04 & -0.37 \\
\hline & 0 & 120 & 38.63 & 38.99 & 0.92 & 3.39 & 3.38 & $\begin{array}{l}-0.27 \\
\end{array}$ & 26.89 & 26.82 & -0.26 \\
\hline & 0 & 150 & 38.03 & 38.09 & 0.16 & 3.35 & 3.34 & $\begin{array}{l}-0.30 \\
\end{array}$ & 26.58 & 26.61 & 0.11 \\
\hline & 1 & 0 & 39.23 & 39.23 & 0.00 & 3.39 & 3.39 & 0.00 & 26.86 & 26.86 & 0.00 \\
\hline & 1 & 30 & 43.23 & 43.23 & 0.00 & 3.56 & 3.56 & 0.00 & 27.75 & 27.75 & 0.00 \\
\hline & 1 & 60 & 40.83 & 41.18 & 0.85 & 3.45 & 3.48 & 0.69 & 27.36 & 27.32 & -0.15 \\
\hline & 1 & 90 & 39.03 & 39.31 & 0.71 & 3.37 & 3.39 & 0.68 & 26.91 & 26.86 & -0.19 \\
\hline & 1 & 120 & 37.23 & 37.60 & 0.98 & 3.31 & 3.32 & 0.15 & 26.47 & 26.40 & -0.27 \\
\hline & 1 & 150 & 36.03 & 36.03 & 0.00 & 3.24 & 3.24 & 0.00 & 26.06 & 26.11 & 0.19 \\
\hline & 3 & 0 & 39.23 & 39.23 & 0.00 & 3.39 & 3.39 & 0.00 & 26.86 & 26.86 & 0.00 \\
\hline & 3 & 30 & 44.53 & 44.57 & 0.09 & 3.61 & 3.61 & 0.00 & 28.03 & 28.02 & -0.04 \\
\hline & 3 & 60 & 40.73 & 41.30 & 1.38 & 3.46 & 3.48 & 0.57 & 27.18 & 27.38 & 0.73 \\
\hline
\end{tabular}

Retrieval Number: D9086049420/2020@BEIESP DOI: 10.35940/ijeat.D9086.049420 Journal Website: www.ijeat.org 
Effect of Remixing \& Intermittent Curing Sequences on Strength of Pavement Quality Concrete

\begin{tabular}{|l|l|l|l|l|l|l|l|l|l|l|l|}
\hline & 3 & 90 & 38.23 & 38.55 & 0.83 & 3.35 & 3.36 & 0.27 & 26.63 & 26.77 & 0.52 \\
\cline { 2 - 11 } & 3 & 120 & 35.73 & 36.13 & 1.11 & 3.28 & 3.25 & -1.02 & 26.03 & 26.18 & 0.57 \\
\cline { 2 - 11 } & 3 & 150 & 33.93 & 33.99 & 0.18 & 3.14 & 3.14 & 0.00 & 25.59 & 25.62 & 0.12 \\
\cline { 2 - 11 } & $\infty$ & 0 & 39.23 & 39.23 & 0.00 & 3.39 & 3.39 & 0.00 & 26.86 & 26.86 & 0.00 \\
\cline { 2 - 12 } & $\infty$ & 30 & 46.03 & 46.03 & 0.00 & 3.67 & 3.67 & 0.00 & 28.35 & 28.35 & 0.00 \\
\cline { 2 - 12 } & $\infty$ & 90 & 34.93 & 35.65 & 2.02 & 3.17 & 3.24 & 2.19 & 26.20 & 26.50 & 1.13 \\
\cline { 2 - 11 } & 120 & 31.33 & 32.01 & 2.12 & 3.02 & 3.06 & 1.34 & 25.37 & 25.66 & 1.13 & 27.39 \\
\cline { 2 - 10 } & 150 & 29.03 & 29.03 & 0.00 & 2.90 & 2.90 & 0.00 & 24.87 & 24.87 & 0.00 \\
\hline
\end{tabular}

Table X: Comparison between the analytical and experimental values of Selfed strength of Mix M40 for different time lag parameters (Type of Curing $A_{07} W_{21}$.

\begin{tabular}{|c|c|c|c|c|c|c|c|c|c|c|c|}
\hline \multirow{2}{*}{$\begin{array}{c}\text { Type } \\
\text { of } \\
\text { Curing }\end{array}$} & \multirow{2}{*}{$\begin{array}{c}\text { Blend } \\
\text { Ratio } \\
\text { (r) }\end{array}$} & \multirow{2}{*}{$\begin{array}{c}\text { Time } \\
\text { Lag } \\
\text { (Min.) } \\
\text { t }\end{array}$} & \multicolumn{3}{|c|}{$\begin{array}{l}\text { Compressive strength } \\
\text { (N/mm2) }\end{array}$} & \multicolumn{3}{|c|}{ Tensile strength(N/mm2) } & \multicolumn{3}{|c|}{$\begin{array}{c}\text { Modulus of Elasticity in } \\
\text { Gpa) }\end{array}$} \\
\hline & & & $\mathrm{XC}(\mathrm{t})$ & $\mathrm{AC}(\mathrm{t})$ & $\begin{array}{c}\% \\
\text { Error }\end{array}$ & $\mathrm{XT}(\mathrm{t})$ & AT(t) & $\begin{array}{c}\% \\
\text { Error }\end{array}$ & $\mathrm{XE}(\mathrm{t})$ & $\mathrm{AE}(\mathrm{t})$ & $\begin{array}{c}\% \\
\text { Error }\end{array}$ \\
\hline \multirow{22}{*}{$\mathbf{A}_{07} \mathbf{W}_{21}$} & 0 & 0 & 42.74 & 42.74 & 0.00 & 3.62 & 3.62 & 0.00 & 28.05 & 28.05 & 0.00 \\
\hline & 0 & 30 & 45.54 & 45.43 & -0.24 & 3.72 & 3.72 & 0.00 & 28.61 & 28.60 & -0.03 \\
\hline & 0 & 60 & 44.44 & 44.51 & 0.16 & 3.68 & 3.68 & 0.05 & 28.52 & 28.40 & -0.42 \\
\hline & 0 & 90 & 43.34 & 43.62 & 0.64 & 3.62 & 3.64 & 0.47 & 28.31 & 28.21 & -0.35 \\
\hline & 0 & 120 & 42.74 & 42.77 & 0.06 & 3.60 & 3.62 & 0.64 & 28.07 & 28.02 & -0.18 \\
\hline & 0 & 150 & 42.74 & 42.77 & 0.06 & 3.60 & 3.62 & 0.64 & 28.07 & 28.02 & -0.18 \\
\hline & 1 & 0 & 42.94 & 42.94 & 0.00 & 3.62 & 3.62 & 0.00 & 28.05 & 28.05 & 0.00 \\
\hline & 1 & 30 & 46.74 & 46.74 & 0.00 & 3.77 & 3.77 & 0.00 & 28.86 & 28.86 & 0.00 \\
\hline & 1 & 60 & 43.94 & 44.91 & 2.16 & 3.70 & 3.64 & -1.71 & 28.48 & 28.48 & 0.00 \\
\hline & 1 & 90 & 42.64 & 43.13 & 1.14 & 3.63 & 3.67 & 1.31 & 28.08 & 28.11 & 0.11 \\
\hline & 1 & 120 & 41.44 & 41.52 & 0.19 & 3.56 & 3.59 & 0.97 & 27.69 & 27.75 & 0.22 \\
\hline & 1 & 150 & 40.04 & 40.04 & 0.00 & 3.49 & 3.49 & 0.00 & 27.40 & 27.40 & 0.00 \\
\hline & 3 & 0 & 42.94 & 42.94 & 0.00 & 3.62 & 3.62 & 0.00 & 28.05 & 28.05 & 0.00 \\
\hline & 3 & 30 & 48.14 & 47.94 & -0.42 & 3.82 & 3.83 & 0.05 & 29.14 & 29.12 & -0.07 \\
\hline & 3 & 60 & 44.64 & 45.11 & 1.04 & 3.69 & 3.71 & 0.67 & 28.41 & 28.55 & 0.49 \\
\hline & 3 & 90 & 41.74 & 42.52 & 1.83 & 3.56 & 3.61 & 1.39 & 27.79 & 28.00 & 0.75 \\
\hline & 3 & 120 & 39.74 & 40.22 & 1.19 & 3.48 & 3.51 & 0.86 & 27.33 & 27.47 & 0.51 \\
\hline & 3 & 150 & 38.14 & 38.15 & 0.03 & 3.42 & 3.41 & -0.12 & 26.95 & 26.97 & 0.07 \\
\hline & $\infty$ & 0 & 42.94 & 42.94 & 0.00 & 3.62 & 3.62 & 0.00 & 28.05 & 28.05 & 0.00 \\
\hline & $\infty$ & 30 & 48.54 & 48.54 & 0.00 & 3.84 & 3.84 & 0.00 & 29.22 & 29.22 & 0.00 \\
\hline & $\infty$ & 60 & 42.14 & 42.74 & 1.40 & 3.54 & 3.61 & 2.02 & 28.02 & 28.28 & 0.92 \\
\hline & $\infty$ & 90 & 38.34 & 38.74 & 1.03 & 3.33 & 3.41 & 2.40 & 27.06 & 27.41 & 1.28 \\
\hline
\end{tabular}

Retrieval Number: D9086049420/2020@BEIESP DOI: $10.35940 /$ ijeat.D9086.049420 Journal Website: www.ijeat.org
Published By:

Blue Eyes Intelligence Engineering \& Sciences Publication 2005 (C) Copyright: All rights reserved. 


\begin{tabular}{|c|c|c|c|c|c|c|c|c|c|c|c|}
\hline & $\infty$ & 120 & 35.74 & 36.52 & 2.14 & 3.19 & 3.23 & 1.30 & 26.37 & 26.58 & 0.79 \\
\cline { 2 - 11 } & $\infty$ & 150 & 33.74 & 33.74 & 0.00 & 3.07 & 3.07 & 0.00 & 25.80 & 25.80 & 0.00 \\
\hline
\end{tabular}

Table XI: Comparison between the analytical and experimental values of Selfed strength of Mix M40 for different time lag parameters (Type of Curing A00W28).

\begin{tabular}{|c|c|c|c|c|c|c|c|c|c|c|c|}
\hline \multirow{2}{*}{$\begin{array}{l}\text { Type of } \\
\text { Curing }\end{array}$} & \multirow{2}{*}{$\begin{array}{c}\text { Blend } \\
\text { Ratio } \\
\text { (r) }\end{array}$} & \multirow{2}{*}{$\begin{array}{c}\text { Time } \\
\text { Lag } \\
\text { (Min.) } \\
\text { t }\end{array}$} & \multicolumn{3}{|c|}{$\begin{array}{c}\text { Compressive strength } \\
\text { (N/mm2) }\end{array}$} & \multicolumn{3}{|c|}{ Tensile strength(N/mm2) } & \multicolumn{3}{|c|}{ Modulus of Elasticity in Gpa) } \\
\hline & & & $\mathrm{XC}(\mathrm{t})$ & $\mathrm{AC}(\mathrm{t})$ & $\begin{array}{c}\% \\
\text { Error }\end{array}$ & $\mathrm{XT}(\mathrm{t})$ & $\operatorname{AT}(\mathbf{t})$ & $\begin{array}{c}\% \\
\text { Error }\end{array}$ & $\mathrm{XE}(\mathrm{t})$ & $\mathrm{AE}(\mathrm{t})$ & $\begin{array}{c}\% \\
\text { Error }\end{array}$ \\
\hline \multirow{24}{*}{$A_{00} W_{28}$} & 0 & 0 & 39.25 & 39.25 & 0.00 & 3.39 & 3.39 & 0.00 & 26.89 & 26.89 & 0.00 \\
\hline & 0 & 30 & 42.05 & 41.99 & -0.14 & 3.51 & 3.51 & 0.00 & 27.52 & 27.51 & -0.04 \\
\hline & 0 & 60 & 40.65 & 40.55 & -0.25 & 3.55 & 3.55 & 0.00 & 27.39 & 27.29 & -0.37 \\
\hline & 0 & 90 & 39.45 & 39.95 & 1.25 & 3.41 & 3.42 & 0.38 & 27.17 & 27.07 & -0.37 \\
\hline & 0 & 120 & 38.65 & 39.01 & 0.92 & 3.39 & 3.38 & -0.27 & 26.92 & 26.85 & -0.26 \\
\hline & 0 & 150 & 38.05 & 38.10 & 0.13 & 3.35 & 3.34 & -0.30 & 26.61 & 26.64 & 0.11 \\
\hline & 1 & 0 & 39.25 & 39.25 & 0.00 & 3.39 & 3.39 & 0.00 & 26.89 & 26.89 & 0.00 \\
\hline & 1 & 30 & 43.25 & 43.25 & 0.00 & 3.56 & 3.56 & 0.00 & 27.78 & 27.80 & 0.07 \\
\hline & 1 & 60 & 40.85 & 41.19 & 0.83 & 3.45 & 3.48 & 0.69 & 27.39 & 27.35 & -0.15 \\
\hline & 1 & 90 & 39.05 & 39.33 & 0.71 & 3.37 & 3.39 & 0.68 & 26.94 & 26.94 & 0.00 \\
\hline & 1 & 120 & 37.25 & 37.62 & 0.98 & 3.31 & 3.32 & 0.15 & 26.50 & 26.53 & 0.11 \\
\hline & 1 & 150 & 36.05 & 36.05 & 0.00 & 3.24 & 3.24 & 0.00 & 26.14 & 26.14 & 0.00 \\
\hline & 3 & 0 & 39.25 & 39.25 & 0.00 & 3.39 & 3.39 & 0.00 & 26.89 & 26.89 & 0.00 \\
\hline & 3 & 30 & 44.55 & 44.49 & -0.13 & 3.61 & 3.61 & 0.00 & 27.96 & 28.05 & 0.32 \\
\hline & 3 & 60 & 40.75 & 41.32 & 1.38 & 3.46 & 3.48 & 0.57 & 27.23 & 27.41 & 0.66 \\
\hline & 3 & 90 & 38.25 & 38.57 & 0.83 & 3.35 & 3.36 & 0.27 & 26.66 & 26.80 & 0.52 \\
\hline & 3 & 120 & 35.75 & 36.15 & 1.11 & 3.23 & 3.25 & 0.52 & 26.06 & 26.21 & 0.57 \\
\hline & 3 & 150 & 34.01 & 34.01 & 0.00 & 3.15 & 3.14 & -0.29 & 25.62 & 25.65 & 0.12 \\
\hline & $\infty$ & 0 & 39.30 & 39.25 & -0.13 & 3.39 & 3.39 & 0.00 & 26.89 & 26.89 & 0.00 \\
\hline & $\infty$ & 30 & 46.05 & 46.05 & 0.00 & 3.67 & 3.67 & 0.00 & 28.38 & 28.38 & 0.00 \\
\hline & $\infty$ & 60 & 38.45 & 39.06 & 1.56 & 3.37 & 3.40 & 0.88 & 26.70 & 27.15 & 1.66 \\
\hline & $\infty$ & 90 & 34.95 & 35.01 & 0.17 & 3.17 & 3.20 & 0.94 & 26.20 & 26.53 & 1.24 \\
\hline & $\infty$ & 120 & 31.35 & 31.65 & 0.94 & 3.02 & 3.06 & 1.34 & 26.00 & 25.69 & -1.21 \\
\hline & $\infty$ & 150 & 29.05 & 29.05 & 0.00 & 2.90 & 2.90 & 0.00 & 24.90 & 24.90 & 0.00 \\
\hline
\end{tabular}

Published By:

Blue Eyes Intelligence Engineering \& Sciences Publication 2006 (C) Copyright: All rights reserved. 
Effect of Remixing \& Intermittent Curing Sequences on Strength of Pavement Quality Concrete

Table XII: Comparison between the analytical and experimental values of Selfed strength of Mix M50 for different time lag parameters (Type of Curing A28W00).

\begin{tabular}{|c|c|c|c|c|c|c|c|c|c|c|c|}
\hline \multirow{3}{*}{$\begin{array}{l}\text { Type of } \\
\text { Curing }\end{array}$} & \multirow{3}{*}{$\begin{array}{c}\text { Blend } \\
\text { Ratio } \\
\text { (r) }\end{array}$} & \multirow{3}{*}{$\begin{array}{c}\text { Time } \\
\text { Lag } \\
\text { (Min.) } \\
\text { t }\end{array}$} & & & & & & & & & \\
\hline & & & \multicolumn{3}{|c|}{$\begin{array}{l}\text { Compressive strength } \\
\text { (N/mm2) }\end{array}$} & \multicolumn{3}{|c|}{ Tensile strength(N/mm2) } & \multicolumn{3}{|c|}{ Modulus of Elasticity in Gpa) } \\
\hline & & & $\mathrm{XC}(\mathrm{t})$ & $\mathrm{AC}(\mathrm{t})$ & $\begin{array}{c}\% \\
\text { Error }\end{array}$ & $\mathrm{XT}(\mathrm{t})$ & $\operatorname{AT}(\mathbf{t})$ & $\begin{array}{c}\% \\
\text { Error }\end{array}$ & $\mathrm{XE}(\mathrm{t})$ & $\mathrm{AE}(\mathrm{t})$ & $\begin{array}{c}\% \\
\text { Error }\end{array}$ \\
\hline \multirow{24}{*}{$A_{28} W_{00}$} & 0 & 0 & 46.75 & 46.75 & 0.00 & 4.04 & 4.04 & 0.00 & 26.82 & 26.82 & 0.00 \\
\hline & 0 & 30 & 51.35 & 51.44 & 0.17 & 4.24 & 4.25 & 0.24 & 27.70 & 27.70 & 0.00 \\
\hline & 0 & 60 & 49.75 & 50.22 & 0.93 & 4.18 & 4.20 & 0.45 & 27.38 & 27.48 & 0.36 \\
\hline & 0 & 90 & 48.45 & 49.04 & 1.21 & 4.13 & 4.15 & 0.34 & 27.20 & 27.26 & 0.22 \\
\hline & 0 & 120 & 47.75 & 47.92 & 0.36 & 4.09 & 4.10 & 0.24 & 27.01 & 27.05 & 0.15 \\
\hline & 0 & 150 & 46.95 & 46.79 & \begin{tabular}{|l|}
-0.34 \\
\end{tabular} & 4.05 & 4.05 & 0.00 & 26.86 & 26.86 & 0.00 \\
\hline & 1 & 0 & 46.75 & 46.75 & 0.00 & 4.04 & 4.04 & 0.00 & 26.82 & 26.82 & 0.00 \\
\hline & 1 & 30 & 52.45 & 52.45 & 0.00 & 4.28 & 4.28 & 0.00 & 27.87 & 27.87 & 0.00 \\
\hline & 1 & 60 & 50.45 & 50.57 & 0.23 & 4.18 & 4.21 & 0.59 & 27.37 & 27.54 & 0.62 \\
\hline & 1 & 90 & 48.25 & 48.82 & 1.16 & 4.10 & 4.13 & 0.92 & 27.11 & 27.23 & 0.44 \\
\hline & 1 & 120 & 47.05 & 47.18 & 0.28 & 4.00 & 4.00 & 0.00 & 26.79 & 26.91 & 0.45 \\
\hline & 1 & 150 & 45.65 & 45.65 & 0.00 & 4.04 & 4.04 & 0.00 & 26.61 & 26.61 & 0.00 \\
\hline & 3 & 0 & 46.75 & 46.75 & 0.00 & 4.04 & 4.04 & 0.00 & 26.82 & 26.82 & 0.00 \\
\hline & 3 & 30 & 53.25 & 53.44 & 0.36 & 4.31 & 4.32 & 0.09 & 28.05 & 28.04 & -0.04 \\
\hline & 3 & 60 & 50.35 & 50.88 & 1.03 & 4.18 & 4.22 & 0.95 & 27.53 & 27.61 & 0.29 \\
\hline & 3 & 90 & 47.85 & 48.54 & 1.42 & 4.09 & 4.12 & 0.85 & 27.01 & 27.18 & 0.63 \\
\hline & 3 & 120 & 45.95 & 46.41 & 0.99 & 4.02 & 4.03 & 0.37 & 26.74 & 26.77 & 0.11 \\
\hline & 3 & 150 & 44.55 & 44.55 & 0.00 & 3.95 & 3.94 & -0.05 & 26.39 & 26.38 & -0.04 \\
\hline & $\infty$ & 0 & 46.75 & 46.75 & 0.00 & 4.04 & 4.04 & 0.00 & 26.82 & 26.82 & 0.00 \\
\hline & $\infty$ & 30 & 47.75 & 48.05 & 0.62 & 4.10 & 4.10 & 0.00 & 27.07 & 27.07 & 0.00 \\
\hline & $\infty$ & 60 & 45.35 & 46.02 & 1.46 & 3.88 & 4.01 & 3.37 & 26.57 & 26.70 & 0.49 \\
\hline & $\infty$ & 90 & 43.45 & 43.85 & 0.91 & 3.90 & 3.93 & 0.87 & 26.17 & 26.34 & 0.65 \\
\hline & $\infty$ & 120 & 41.95 & 42.44 & 1.15 & 3.86 & 3.85 & -0.13 & 26.47 & 25.98 & -1.89 \\
\hline & $\infty$ & 150 & 40.85 & 40.85 & 0.00 & 3.78 & 3.78 & 0.00 & 25.74 & 25.74 & 0.00 \\
\hline
\end{tabular}

Table XIII: Comparison between the analytical and experimental values of Selfed strength of Mix M50 for different time lag parameters (Type of Curing A21W07).

\begin{tabular}{|c|c|c|c|c|c|c|c|c|c|c|c|}
\hline \multirow{2}{*}{$\begin{array}{c}\text { Type } \\
\text { of } \\
\text { Curing }\end{array}$} & \multirow{2}{*}{$\begin{array}{c}\text { Blend } \\
\text { Ratio } \\
\text { (r) }\end{array}$} & \multirow{2}{*}{$\begin{array}{c}\text { Time } \\
\text { Lag } \\
\text { (Min.) } \\
\text { t }\end{array}$} & \multicolumn{3}{|c|}{$\begin{array}{l}\text { Compressive strength } \\
\text { (N/mm2) }\end{array}$} & \multicolumn{3}{|c|}{ Tensile strength(N/mm2) } & \multicolumn{3}{|c|}{$\begin{array}{c}\text { Modulus of Elasticity in } \\
\text { Gpa) }\end{array}$} \\
\hline & & & $\mathrm{XC}(\mathrm{t})$ & $A C(t)$ & $\begin{array}{c}\% \\
\text { Error }\end{array}$ & $X T(t)$ & $\operatorname{AT}(t)$ & $\begin{array}{c}\% \\
\text { Error }\end{array}$ & $\mathrm{XE}(\mathrm{t})$ & $\mathrm{AE}(\mathrm{t})$ & $\begin{array}{c}\% \\
\text { Error }\end{array}$ \\
\hline \multirow{2}{*}{$\mathbf{A}_{21} \mathbf{W}_{07}$} & 0 & 0 & 55.67 & 55.67 & 0.00 & 4.41 & 4.41 & 0.00 & 31.62 & 31.62 & 0.00 \\
\hline & 0 & 30 & 56.67 & 56.60 & -0.12 & 4.45 & 4.45 & -0.07 & 31.82 & 31.81 & -0.03 \\
\hline
\end{tabular}

Retrieval Number: D9086049420/2020@BEIESP DOI: $10.35940 /$ ijeat.D9086.049420 Journal Website: www.ijeat.org
Published By:

Blue Eyes Intelligence Engineering \& Sciences Publication 2007 (C) Copyright: All rights reserved. 


\begin{tabular}{|c|c|c|c|c|c|c|c|c|c|c|}
\hline 0 & 60 & 56.57 & 56.20 & -0.65 & 4.44 & 4.43 & -0.25 & 31.80 & 31.73 & -0.22 \\
\hline 0 & 90 & 55.67 & 55.67 & 0.00 & 4.43 & 4.41 & -0.39 & 31.72 & 31.64 & -0.25 \\
\hline 0 & 120 & 55.42 & 55.47 & 0.09 & 4.34 & 4.36 & 0.44 & 31.66 & 31.92 & 0.81 \\
\hline 0 & 150 & 54.87 & 54.77 & -0.18 & 4.27 & 4.27 & 0.00 & 31.47 & 31.47 & 0.00 \\
\hline 1 & 0 & 55.67 & 55.67 & 0.00 & 4.41 & 4.41 & 0.00 & 31.62 & 31.62 & 0.00 \\
\hline 1 & 30 & 57.67 & 57.54 & -0.22 & 4.49 & 4.48 & -0.11 & 32.01 & 31.99 & -0.06 \\
\hline 1 & 60 & 56.77 & 56.66 & -0.18 & 4.45 & 4.45 & -0.09 & 31.84 & 31.82 & -0.06 \\
\hline 1 & 90 & 55.37 & 55.63 & 0.47 & 4.42 & 4.41 & -0.23 & 31.66 & 31.62 & -0.13 \\
\hline 1 & 120 & 54.87 & 54.63 & -0.43 & 4.38 & 4.37 & -0.23 & 31.47 & 31.42 & -0.16 \\
\hline 1 & 150 & 53.67 & 53.67 & 0.00 & 4.83 & 4.33 & -11.56 & 31.23 & 31.23 & 0.00 \\
\hline 3 & 0 & 55.67 & 55.67 & 0.00 & 4.41 & 4.41 & 0.00 & 31.62 & 31.62 & 0.00 \\
\hline 3 & 30 & 57.67 & 58.37 & 1.20 & 4.52 & 4.56 & 0.92 & 2.20 & 32.16 & 93.16 \\
\hline 3 & 60 & 56.37 & 56.91 & 0.95 & 4.44 & 4.46 & 0.49 & 31.95 & 31.91 & -0.13 \\
\hline 3 & 90 & 54.87 & 55.50 & 1.15 & 4.39 & 4.40 & 0.25 & 31.66 & 31.60 & -0.19 \\
\hline 3 & 120 & 53.87 & 54.00 & 0.24 & 4.33 & 4.34 & 0.41 & 31.33 & 31.31 & -0.06 \\
\hline 3 & 150 & 52.67 & 52.57 & -0.18 & 4.29 & 4.29 & 0.00 & 31.03 & 31.02 & -0.03 \\
\hline$\infty$ & 0 & 55.67 & 55.67 & 0.00 & 4.41 & 4.41 & 0.00 & 31.62 & 31.62 & 0.00 \\
\hline$\infty$ & 30 & 58.97 & 58.75 & -0.37 & 4.54 & 4.53 & -0.18 & 32.25 & 32.21 & -0.12 \\
\hline$\infty$ & 60 & 55.67 & 56.15 & 0.86 & 4.40 & 4.43 & 0.77 & 31.56 & 31.74 & 0.57 \\
\hline$\infty$ & 90 & 52.47 & 53.41 & 1.77 & 4.29 & 4.32 & 0.72 & 31.19 & 31.21 & 0.06 \\
\hline$\infty$ & 120 & 50.37 & 50.93 & 1.11 & 4.20 & 4.22 & 0.55 & 30.56 & 30.70 & 0.46 \\
\hline$\infty$ & 150 & 48.67 & 48.67 & 0.00 & 4.12 & 4.12 & 0.00 & 30.21 & 30.21 & 0.00 \\
\hline
\end{tabular}

Table XIV: Comparison between the analytical and experimental values of Selfed strength of Mix M50 for different time lag parameters (Type of Curing A14W14).

\begin{tabular}{|c|c|c|c|c|c|c|c|c|c|c|c|}
\hline \multirow{2}{*}{$\begin{array}{c}\text { Type } \\
\text { of } \\
\text { Curing }\end{array}$} & \multirow{2}{*}{$\begin{array}{c}\text { Blend } \\
\text { Ratio } \\
\text { (r) }\end{array}$} & \multirow{2}{*}{$\begin{array}{c}\text { Time } \\
\text { Lag } \\
\text { (Min.) } \\
\text { t }\end{array}$} & \multicolumn{3}{|c|}{$\begin{array}{c}\text { Compressive strength } \\
\text { (N/mm2) }\end{array}$} & \multicolumn{3}{|c|}{ Tensile strength(N/mm2) } & \multicolumn{3}{|c|}{$\begin{array}{l}\text { Modulus of Elasticity in } \\
\text { Gpa) }\end{array}$} \\
\hline & & & $\mathrm{XC}(\mathrm{t})$ & $\mathrm{AC}(\mathrm{t})$ & $\begin{array}{c}\% \\
\text { Error }\end{array}$ & $X T(t)$ & $\operatorname{AT}(t)$ & $\begin{array}{l}\% \\
\text { Error }\end{array}$ & $X E(t)$ & $\mathrm{AE}(\mathrm{t})$ & $\begin{array}{c}\% \\
\text { Error }\end{array}$ \\
\hline \multirow{8}{*}{$\mathrm{A}_{14} \mathrm{~W}_{14}$} & 0 & 0 & 57.95 & 57.95 & 0.00 & 4.43 & 4.43 & 0.00 & 31.84 & 31.84 & 0.00 \\
\hline & 0 & 30 & 58.55 & 58.60 & 0.09 & 4.46 & 4.46 & -0.04 & 31.95 & 31.96 & 0.03 \\
\hline & 0 & 60 & 58.75 & 58.57 & -0.31 & 4.47 & 4.46 & -0.16 & 31.98 & 31.95 & -0.09 \\
\hline & 0 & 90 & 58.85 & 58.50 & -0.60 & 4.47 & 4.46 & -0.34 & 32.00 & 31.94 & -0.19 \\
\hline & 0 & 120 & 57.65 & 58.42 & 1.32 & 4.46 & 4.45 & -0.04 & 31.95 & 31.92 & -0.09 \\
\hline & 0 & 150 & 58.35 & 58.35 & 0.00 & 4.43 & 4.43 & 0 & 31.90 & 31.90 & 0.00 \\
\hline & 1 & 0 & 57.95 & 57.95 & 0.00 & 4.43 & 4.43 & 0.00 & 31.84 & 31.84 & 0.00 \\
\hline & 1 & 30 & 59.35 & 59.29 & -0.10 & 4.49 & 4.48 & -0.07 & 32.10 & 32.07 & -0.09 \\
\hline $\begin{array}{l}\text { Retrieval } \\
\text { DOI: } 10.3 \\
\text { Journal W }\end{array}$ & $\begin{array}{l}\text { aber: D90 } \\
\text { Olijeat.DS } \\
\text { /ite: www. }\end{array}$ & 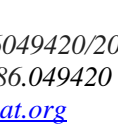 & & & & 008 () & $\begin{array}{l}\text { shed By: } \\
\text { Eyes Inte } \\
\text { iences Pu } \\
\text { pyright: }\end{array}$ & $\begin{array}{l}\text { gence Engin } \\
\text { cation } \\
\text { rights reser }\end{array}$ & & & \\
\hline
\end{tabular}


Effect of Remixing \& Intermittent Curing Sequences on Strength of Pavement Quality Concrete

\begin{tabular}{|l|l|l|l|l|l|l|l|l|l|l|l|}
\hline & 1 & 60 & 58.75 & 58.71 & -0.07 & 4.47 & 4.46 & -0.09 & 31.98 & 31.98 & 0.00 \\
\hline 1 & 90 & 58.15 & 58.04 & -0.19 & 4.44 & 4.44 & -0.11 & 31.87 & 31.85 & -0.06 \\
\hline 1 & 120 & 57.75 & 57.39 & -0.63 & 4.42 & 4.41 & -0.23 & 31.80 & 31.71 & -0.28 \\
\hline 1 & 150 & 56.75 & 56.75 & 0.00 & 4.39 & 4.39 & 0.00 & 31.60 & 31.60 & 0.00 \\
\hline 3 & 0 & 57.95 & 57.95 & 0.00 & 4.46 & 4.43 & -0.74 & 31.84 & 31.84 & 0.00 \\
\hline 3 & 30 & 59.95 & 59.91 & -0.07 & 4.51 & 4.51 & -0.11 & 32.21 & 32.20 & -0.03 \\
\hline 3 & 60 & 58.45 & 58.91 & 0.78 & 4.47 & 4.46 & -0.16 & 32.02 & 32.00 & -0.06 \\
\hline 3 & 90 & 57.35 & 57.54 & 0.33 & 4.43 & 4.42 & -0.34 & 31.84 & 31.77 & -0.22 \\
\hline 3 & 120 & 56.05 & 56.32 & 0.48 & 4.38 & 4.37 & -0.30 & 32.57 & 32.53 & -0.12 \\
\hline 3 & 150 & 55.15 & 55.16 & 0.02 & 44.60 & 44.60 & 0.00 & 31.30 & 31.30 & 0.00 \\
\hline$\infty$ & 0 & 57.95 & 57.95 & 0.00 & 4.43 & 4.43 & 0.00 & 31.84 & 31.84 & 0.00 \\
\hline$\infty$ & 30 & 60.85 & 60.66 & -0.31 & 4.54 & 4.53 & -0.15 & 32.37 & 32.34 & -0.09 \\
\hline$\infty$ & 60 & 58.15 & 58.83 & 1.16 & 4.48 & 4.47 & -0.20 & 32.06 & 32.01 & -0.16 \\
\hline$\infty$ & 90 & 55.95 & 56.81 & 1.51 & 4.38 & 4.39 & 0.23 & 30.75 & 31.64 & 2.81 \\
\hline$\infty$ & 120 & 54.55 & 54.92 & 0.67 & 4.32 & 4.32 & 0.02 & 31.26 & 31.27 & 0.03 \\
\hline
\end{tabular}

Table XV: Comparison between the analytical and experimental values of Selfed strength of Mix M50 for different time lag parameters (Type of Curing A07W21).

\begin{tabular}{|c|c|c|c|c|c|c|c|c|c|c|c|}
\hline \multirow{2}{*}{$\begin{array}{l}\text { Type of } \\
\text { Curing }\end{array}$} & \multirow{2}{*}{$\begin{array}{c}\text { Blend } \\
\text { Ratio } \\
\text { (r) }\end{array}$} & \multirow{2}{*}{$\begin{array}{c}\text { Time } \\
\text { Lag } \\
\text { (Min.) } \\
\text { t }\end{array}$} & \multicolumn{3}{|c|}{$\begin{array}{l}\text { Compressive strength } \\
\text { (N/mm2) }\end{array}$} & \multicolumn{3}{|c|}{ Tensile strength(N/mm2) } & \multicolumn{3}{|c|}{ Modulus of Elasticity in Gpa) } \\
\hline & & & $\mathrm{XC}(\mathrm{t})$ & $A C(t)$ & $\begin{array}{c}\% \\
\text { Error }\end{array}$ & $X T(t)$ & AT(t) & $\begin{array}{c}\% \\
\text { Error }\end{array}$ & $\mathrm{XE}(\mathrm{t})$ & $\mathrm{AE}(\mathrm{t})$ & $\begin{array}{c}\% \\
\text { Error }\end{array}$ \\
\hline \multirow{15}{*}{$A_{07} W_{21}$} & 0 & 0 & 57.01 & 57.01 & 0.00 & 4.49 & 4.49 & 0.00 & 31.94 & 31.94 & 0.00 \\
\hline & 0 & 30 & 58.01 & 57.96 & -0.09 & 4.53 & 4.52 & -0.04 & 32.13 & 32.12 & -0.03 \\
\hline & 0 & 60 & 58.37 & 58.01 & $\begin{array}{l}-0.62 \\
\end{array}$ & 4.54 & 4.53 & \begin{tabular}{|l|}
-0.33 \\
\end{tabular} & 32.20 & 32.13 & $\begin{array}{l}-0.22 \\
\end{array}$ \\
\hline & 0 & 90 & 58.41 & 58.03 & -0.65 & 4.55 & 4.53 & -0.44 & 32.24 & 32.13 & -0.34 \\
\hline & 0 & 120 & 58.31 & 58.05 & -0.45 & 4.54 & 4.53 & -0.33 & 32.20 & 32.13 & -0.22 \\
\hline & 0 & 150 & 57.01 & 57.01 & 0.00 & 4.49 & 4.49 & 0.00 & 31.94 & 31.94 & 0.00 \\
\hline & 1 & 0 & 57.51 & 58.41 & 1.54 & 4.55 & 4.54 & -0.09 & 32.22 & 32.22 & 0.00 \\
\hline & 1 & 30 & 57.61 & 57.89 & 0.48 & 4.54 & 4.52 & -0.29 & 32.18 & 32.11 & -0.22 \\
\hline & 1 & 60 & 56.81 & 57.26 & 0.78 & 4.52 & 4.50 & -0.38 & 32.07 & 31.99 & -0.25 \\
\hline & 1 & 90 & 56.61 & 56.63 & 0.03 & 4.49 & 4.47 & $\begin{array}{l}-0.36 \\
\end{array}$ & 31.04 & 31.87 & 2.60 \\
\hline & 1 & 120 & 56.01 & 56.01 & 0.00 & 4.45 & 4.45 & 0.00 & 31.75 & 31.75 & 0.00 \\
\hline & 1 & 150 & 57.01 & 57.01 & 0.00 & 4.49 & 4.49 & 0.00 & 31.94 & 31.94 & 0.00 \\
\hline & 3 & 0 & 59.01 & 58.97 & -0.07 & 4.57 & 4.56 & -0.11 & 32.31 & 32.28 & -0.09 \\
\hline & 3 & 30 & 57.41 & 57.74 & 0.57 & 4.50 & 4.52 & 0.40 & 2.10 & 2.08 & -0.96 \\
\hline & 3 & 60 & 56.21 & 56.45 & 0.42 & 4.46 & 4.47 & 0.34 & 31.78 & 31.84 & 0.19 \\
\hline
\end{tabular}

Retrieval Number: D9086049420/2020@BEIESP DOI: $10.35940 /$ ijeat.D9086.049420 Journal Website: www.ijeat.org
Published By:

Blue Eyes Intelligence Engineering \& Sciences Publication 2009 (C) Copyright: All rights reserved. 


\begin{tabular}{|l|l|l|l|l|l|l|l|l|l|l|l|}
\hline \multirow{6}{*}{3} & 90 & 54.51 & 55.20 & 1.25 & 4.34 & 4.42 & 1.85 & 31.65 & 31.60 & -0.16 \\
\cline { 2 - 11 } & 3 & 120 & 54.01 & 54.01 & 0.00 & 4.38 & 4.38 & 0.02 & 31.36 & 31.37 & 0.03 \\
\cline { 2 - 11 } & 3 & 150 & 57.01 & 57.01 & 0.00 & 4.49 & 4.49 & 0.00 & 31.94 & 31.94 & 0.00 \\
\cline { 2 - 11 } & $\infty$ & 0 & 60.11 & 59.88 & -0.38 & 4.61 & 4.60 & -0.17 & 32.51 & 32.47 & -0.12 \\
\cline { 2 - 11 } & $\infty$ & 30 & 56.51 & 57.21 & 1.22 & 4.45 & 4.50 & 1.27 & 31.82 & 32.02 & 0.62 \\
\cline { 2 - 11 } & $\infty$ & 60 & 53.61 & 54.57 & 1.76 & 4.36 & 4.40 & 0.84 & 31.27 & 31.50 & 0.73 \\
\cline { 2 - 11 } & $\infty$ & 120 & 49.81 & 49.81 & 0.00 & 4.20 & 4.20 & 0.00 & 30.52 & 30.52 & 0.00 \\
\cline { 2 - 10 } & $\infty$ & 150 & 49.81 & 49.81 & 0.00 & 4.20 & 4.20 & 0.00 & 30.52 & 30.52 & 0.00 \\
\hline
\end{tabular}

Table XVI: Comparison between the analytical and experimental values of Selfed strength of Mix M50 for different time lag parameters (Type of Curing A00W28).

\begin{tabular}{|c|c|c|c|c|c|c|c|c|c|c|c|}
\hline \multirow{2}{*}{$\begin{array}{c}\text { Type } \\
\text { of } \\
\text { Curing }\end{array}$} & \multirow{2}{*}{$\begin{array}{c}\text { Blend } \\
\text { Ratio } \\
\text { (r) }\end{array}$} & \multirow{2}{*}{$\begin{array}{c}\text { Time } \\
\text { Lag } \\
\text { (Min.) } \\
\text { t }\end{array}$} & \multicolumn{3}{|c|}{$\begin{array}{l}\text { Compressive strength } \\
\text { (N/mm2) }\end{array}$} & \multicolumn{3}{|c|}{$\begin{array}{c}\text { Tensile } \\
\text { strength(N/mm2) }\end{array}$} & \multicolumn{3}{|c|}{$\begin{array}{l}\text { Modulus of Elasticity in } \\
\text { Gpa) }\end{array}$} \\
\hline & & & $X C(t)$ & $A C(t)$ & $\begin{array}{c}\% \\
\text { Error }\end{array}$ & $X T(t)$ & $\mathbf{A T}(\mathbf{t})$ & $\begin{array}{c}\% \\
\text { Error }\end{array}$ & $X E(t)$ & $\mathrm{AE}(\mathrm{t})$ & $\begin{array}{c}\% \\
\text { Error }\end{array}$ \\
\hline \multirow{22}{*}{$A_{00} W_{28}$} & 0 & 0 & 55.91 & 55.91 & 0.00 & 3.67 & 3.67 & 0.00 & 31.42 & 31.42 & 0.00 \\
\hline & 0 & 30 & 56.61 & 56.62 & 0.02 & 3.70 & 3.70 & -0.05 & 31.55 & 31.55 & 0.00 \\
\hline & 0 & 60 & 56.91 & 55.61 & -2.34 & 3.70 & 3.70 & -0.14 & 31.61 & 31.55 & -0.19 \\
\hline & 0 & 90 & 57.01 & 56.56 & $\begin{array}{l}-0.79 \\
\end{array}$ & 3.72 & 3.70 & -0.54 & 31.63 & 31.54 & $\begin{array}{l}-0.29 \\
\end{array}$ \\
\hline & 0 & 120 & 57.01 & 56.53 & -0.85 & 3.71 & 3.70 & -0.27 & 31.61 & 31.53 & -0.25 \\
\hline & 0 & 150 & 56.61 & 56.61 & 0.00 & 3.70 & 3.70 & 0.00 & 31.55 & 31.52 & -0.10 \\
\hline & 1 & 0 & 55.91 & 55.91 & 0.00 & 3.67 & 3.67 & 0.00 & 31.42 & 31.42 & 0.00 \\
\hline & 1 & 30 & 57.41 & 57.31 & -0.17 & 3.78 & 3.78 & -0.05 & 31.70 & 31.68 & -0.06 \\
\hline & 1 & 60 & 56.61 & 56.79 & 0.32 & 3.64 & 3.68 & 1.17 & 31.65 & 31.59 & $\begin{array}{l}-0.19 \\
\end{array}$ \\
\hline & 1 & 90 & 55.81 & 56.15 & 0.61 & 3.54 & 3.57 & 0.95 & 31.55 & 31.47 & -0.25 \\
\hline & 1 & 120 & 55.31 & 55.52 & 0.38 & 3.46 & 3.47 & 0.20 & 31.50 & 31.35 & -0.48 \\
\hline & 1 & 150 & 54.91 & 54.91 & 0.00 & 3.37 & 3.37 & 0.00 & 31.21 & 31.23 & 0.06 \\
\hline & 3 & 0 & 55.91 & 55.91 & 0.00 & 3.67 & 3.67 & 0.00 & 31.42 & 31.42 & 0.00 \\
\hline & 3 & 30 & 58.11 & 58.01 & -0.17 & 3.75 & 3.74 & -0.13 & 31.79 & 31.82 & 0.09 \\
\hline & 3 & 60 & 56.11 & 56.95 & 1.47 & 3.72 & 3.71 & -0.46 & 31.65 & 31.63 & -0.06 \\
\hline & 3 & 90 & 55.21 & 55.27 & 0.11 & 3.65 & 3.66 & 0.27 & 31.28 & 31.39 & 0.35 \\
\hline & 3 & 120 & 54.51 & 54.55 & 0.07 & 3.63 & 3.53 & -2.78 & 31.61 & 31.17 & -1.41 \\
\hline & 3 & 150 & 53.61 & 53.42 & $\begin{array}{l}-0.36 \\
\end{array}$ & 3.48 & 3.48 & 0.00 & 31.47 & 30.94 & -1.71 \\
\hline & $\infty$ & 0 & 55.91 & 55.91 & 0.00 & 3.67 & 3.67 & 0.00 & 31.42 & 31.42 & 0.00 \\
\hline & $\infty$ & 30 & 58.91 & 58.71 & -0.34 & 3.78 & 3.78 & $\begin{array}{l}-0.19 \\
\end{array}$ & 31.98 & 31.94 & -0.13 \\
\hline & $\infty$ & 60 & 55.11 & 56.01 & 1.61 & 3.64 & 3.68 & 1.11 & 31.26 & 31.47 & 0.67 \\
\hline & $\infty$ & 90 & 52.41 & 53.36 & 1.78 & 3.54 & 3.57 & 0.95 & 30.76 & 30.95 & 0.61 \\
\hline $\begin{array}{l}\text { Retrieval Nu } \\
\text { DOI: } 10.359 \\
\text { Journal Web }\end{array}$ & $\begin{array}{l}r: D 90 \varepsilon \\
\text { jeat.D9 } \\
w w w . i\end{array}$ & $\begin{array}{l}49420 / 20 . \\
\text { s.049420 } \\
\text { t.org }\end{array}$ & & & & 010 (잉 & ences Pu & $\begin{array}{l}\text { cation } \\
\text { rights res }\end{array}$ & & & \\
\hline
\end{tabular}


Effect of Remixing \& Intermittent Curing Sequences on Strength of Pavement Quality Concrete

\begin{tabular}{|l|l|l|l|l|l|l|l|l|l|l|l|}
\hline & $\infty$ & 120 & 50.51 & 50.87 & 0.71 & 3.44 & 3.47 & 0.78 & 30.35 & 30.44 & 0.30 \\
\cline { 2 - 11 } & $\infty$ & 150 & 48.61 & 48.61 & 0.00 & 3.37 & 3.37 & 0.00 & 29.95 & 29.95 & 0.00 \\
\hline
\end{tabular}

Table XVII: Intermittent curing effect (Grade M40)

\begin{tabular}{|c|c|c|c|c|c|c|}
\hline $\begin{array}{l}\text { Sr.no./curing } \\
\text { condition }\end{array}$ & Blend ratio (r) & $\begin{array}{l}\text { Cube } \\
\text { Specification }\end{array}$ & $\begin{array}{l}\operatorname{Air}_{21} \\
\text { Water }_{7}\end{array}$ & $\begin{array}{l}\text { Air }_{7} \\
\text { Water21 }\end{array}$ & $\begin{array}{l}\text { Air28 } \\
\text { Watero }\end{array}$ & $\begin{array}{l}\text { Airo } \\
\text { Water28 }\end{array}$ \\
\hline 1 & $r=0$ & $\mathrm{CB}_{1} \mathrm{~T}_{0}$ & 28.65 & 32.2 & 41.96 & 46.8 \\
\hline 2 & \multirow{4}{*}{$r=1$} & $\mathrm{CB}_{1} \mathrm{~T}_{1}$ & 30.51 & 31.56 & 42.89 & 44.56 \\
\hline 3 & & $\mathrm{CB}_{1} \mathrm{~T}_{2}$ & 27.89 & 29.48 & 41.32 & 43.15 \\
\hline 4 & & $\mathrm{CB}_{1} \mathrm{~T}_{3}$ & 25.32 & 27.31 & 39.15 & 41.57 \\
\hline 5 & & $\mathrm{CB}_{1} \mathrm{~T}_{4}$ & 20.57 & 23.18 & 34.15 & 38.15 \\
\hline 6 & \multirow{4}{*}{$r=3$} & $\mathrm{CB}_{3} \mathrm{~T}_{1}$ & 27.98 & 30.56 & 38.5 & 42.32 \\
\hline 7 & & $\mathrm{CB}_{3} \mathrm{~T}_{2}$ & 25.47 & 29.02 & 36.89 & 41.62 \\
\hline 8 & & $\mathrm{CB}_{3} \mathrm{~T}_{3}$ & 24.98 & 26.96 & 35.46 & 40.65 \\
\hline 9 & & $\mathrm{CB}_{3} \mathrm{~T}_{4}$ & 20.57 & 22.1 & 34.15 & 37.34 \\
\hline 10 & \multirow{4}{*}{$\mathrm{r}=$ Infinity } & $\mathrm{CB}_{\text {Infinity }} \mathrm{T}_{1}$ & 29.56 & 30.12 & 40.16 & 42.12 \\
\hline 11 & & $\mathrm{CB}_{\text {infinity }} \mathrm{T}_{2}$ & 28.34 & 29.15 & 38.65 & 39.05 \\
\hline 12 & & $\mathrm{CB}_{\text {infinity }} \mathrm{T}_{3}$ & 24 & 24.31 & 36.41 & 37.52 \\
\hline 13 & & $\mathrm{CB}_{\text {infinity }} \mathrm{T}_{4}$ & 22.05 & 22.15 & 33.42 & 34.62 \\
\hline
\end{tabular}

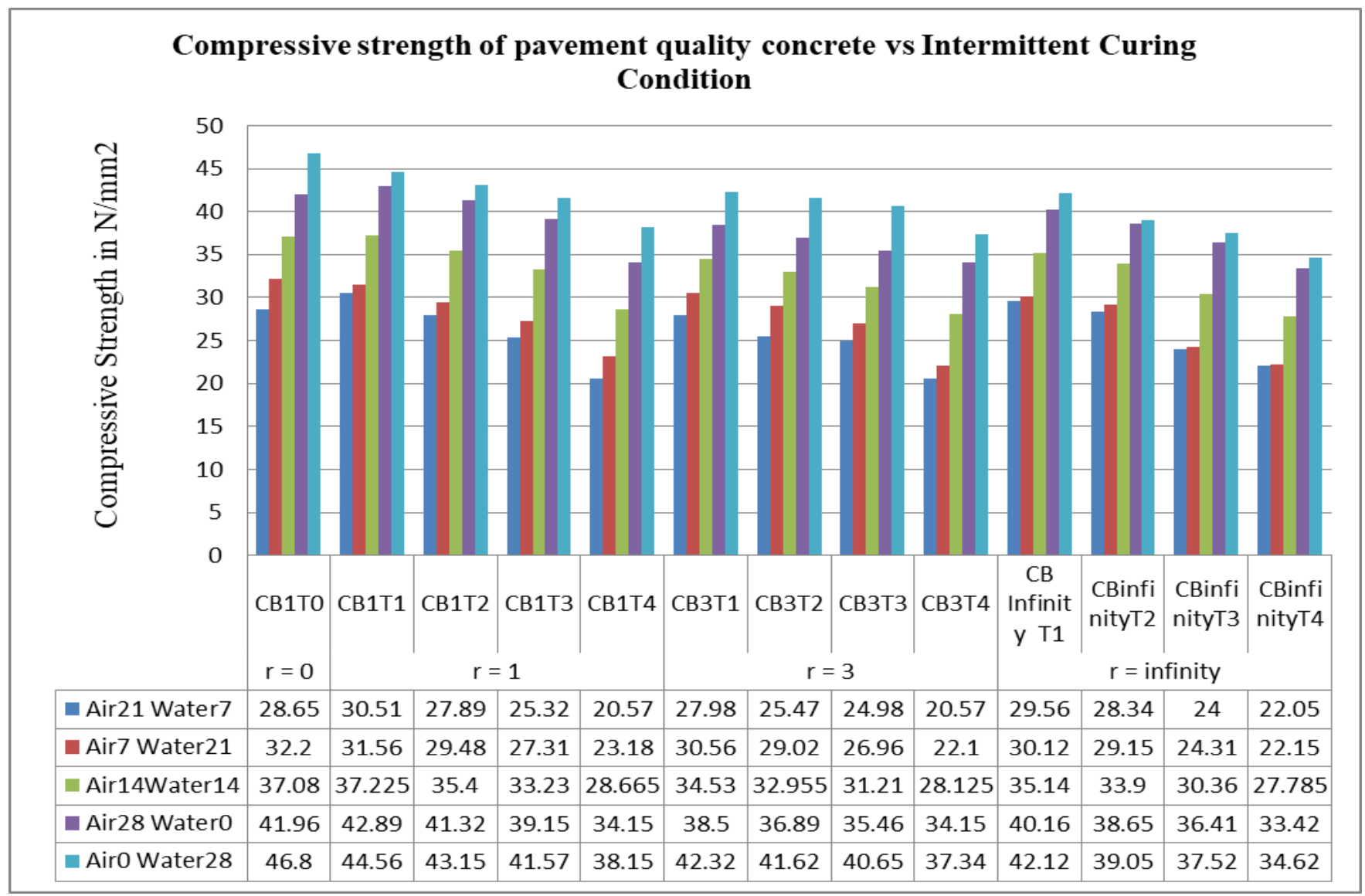

Fig 3 . Compressive strength of pavement quality concrete vs Intermittent Curing Condition \& Blend ratio

Retrieval Number: D9086049420/2020@BEIESP DOI: $10.35940 /$ ijeat.D9086.049420

Journal Website: www.ijeat.org
Published By:

Blue Eyes Intelligence Engineering \& Sciences Publication

2011 (C) Copyright: All rights reserved.

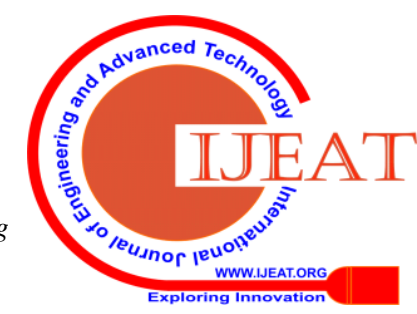




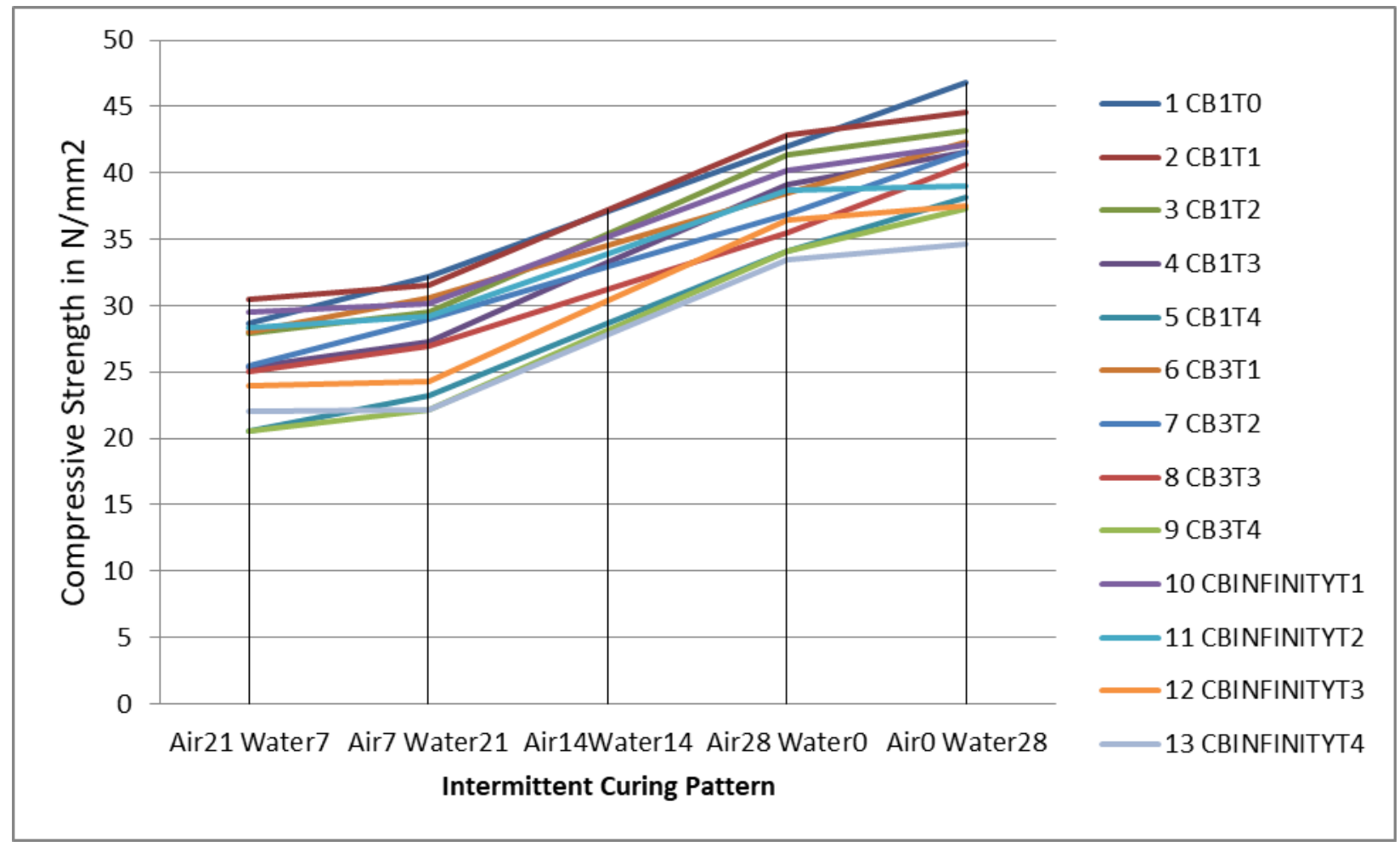

Fig.4 Compressive strength of pavement quality concrete vs Intermittent Curing Condition

\section{Notation:}

$\mathrm{CB}_{1} \mathrm{~T}_{\mathbf{0}}$

$\mathrm{CB}_{1} \mathrm{~T}_{1}$

$\mathrm{CB}_{1} \mathrm{~T}_{2}$

$\mathrm{CB}_{1} \mathrm{~T}_{3}$

$\mathrm{CB}_{1} \mathrm{~T}_{4}$

Similarly

$\mathbf{C B}_{3} \mathbf{T}_{1}$
: $\quad \mathrm{CB}_{1}=$ Casting of Cubes for blend ratio $\mathrm{r}=1, \& \mathrm{~T}_{0}=$ Time lag is 0 Minute
: $\quad \mathrm{CB}_{1}=$ Casting of Cubes for blend ratio $\mathrm{r}=1, \& \mathrm{~T}_{1}=$ Time lag is 30 Minute
: $\quad \mathrm{CB}_{1}=$ Casting of Cubes for blend ratio $\mathrm{r}=1, \& \mathrm{~T}_{2}=$ Time lag is 60 Minute
: $\quad \mathrm{CB}_{1}=$ Casting of Cubes for blend ratio $\mathrm{r}=1, \& \mathrm{~T}_{3}=$ Time lag is 90 Minute
: $\quad \mathrm{CB}_{1}=$ Casting of Cubes for blend ratio $\mathrm{r}=1, \& \mathrm{~T}_{4}=$ Time lag is 120 Minute

: $\mathrm{CB}_{3}=$ Casting of Cubes for blend ratio $\mathrm{r}=3, \& \mathrm{~T}_{1}=$ Time lag is 30 Minute
The observations of the workability parameters such as slump, Vee Bee time and compaction factor recorded at initial as well as final setting times for the basic as well as pure selfed mixes for the various blend ratios, viz., $\mathrm{r}=\infty$, 3.00, 1.00 and 0 are presented in Table XVII. The compressive strength, tensile strength, and modulus of elasticity values calculated from the experimental observations and the effect of improper curing sequences are presented in tables VII to XVI. In tables those tables the experimental values and analytical values of the strengths viz., compressive strength $\mathrm{XC}, \mathrm{AC}$, tensile strength $\mathrm{XT}$, AT and modulus of elasticity XE, AE for all the different mix cases (M40 \& M50), each observed at different time lags t (hr) (viz., 0.00. 30.00, 60.00, 90.00, 120.00 and $150.00 \mathrm{~min}$ ) for each blend ratio $\mathrm{r}(=\infty, 3,1$ and 0$)$ cured under different improper curing sequences WI AII. (Viz., A0W28, A7W21, A21W7, A28W00 W28A00) have been presented.

\section{CONCLUSION}

1 The strength of concrete which undergoes intermittent curing is less than that of strength due to cumulative curing after 28 days of curing.

2 The maximum compressive strength of concrete achieved by using A00W28 Curing Condition.

3 The strength of blended concrete gives better result up to $\mathrm{r}$ $=0,1, \&$ in-between $1 \& 3$. This strength gives satisfactory results up to time lag 90 minutes.
4 Blend Concrete with $r=3$ and infinity gives poor strength so in practice old concrete was not suitable for reuse. As time lag is increased after IST, the concrete becomes dry and set, hence it is preferred to use the concrete before final setting the time.

\section{REFERENCES}

1 Bairagi, N. K., A. S. Goyal, and P. A. Joshi. "Strength of Composite Mixes Using General Crossing Theory." Indian Concrete Journal 63, no. 12 (1989), pp 600-605.

2 N.K.Bairagi,A.S.Goyal\&P.A.Joshi"Strength of composite mixes under cumulative curing using selfing and crossing theory " The Indian Concrete Journal Vol.64 (11) (1990 ), pp 527-538.

3 Fathollah, Sajedi, Hashim, Abdul Razak, "Effects of curing regimes and cement fineness on the compressive strength of ordinary Portland cement mortars." Construction and Building Materials, Vol.25(2011), pp 2036-2045.

4 Zain M.F.M "Effect of different curing methods on the Properties of micro silica concrete". Australian Journal of Basic and Applied Science, Vol.1 (2) (2007),pp 87 -95.

5 N. R. El-Sakhawy, H. S. El-Dien "Influence of curing on durability performance of concrete", Magazine of Concrete Research, Vol.51(1999), pp 309-318.

6 Akeem Ayinde Raheem,and Aliu Adebayo Soyingb, "Effect of curing on density and compressive strength of concrete", International Journal of Applied Science And Technology, Vol.3(2013), pp 56-64.

7 Suresh B Piplewar, Narendra M Kanhe and Devendra Pandey "Intermittent curing of M20 concrete", International Journal of Structure and Civil Engginering, Vol. 2(2013),pp 165-171.

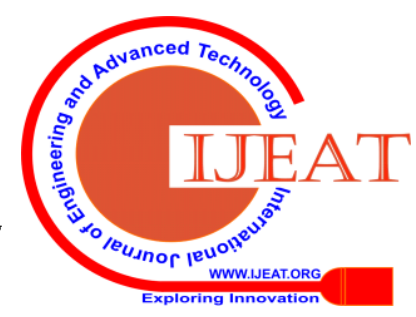


8 Wang, Kejin, James K. Cable, and Zhi Ge. "Evaluation of pavement curing effectiveness and curing effects on concrete properties." Journal of Materials in Civil Engineering 18.3 (2006),pp 377-389.

9 Bentz, Dale P., and W. Jason Weiss. Internal curing: a 2010 state-of-the-art review. Gaithersburg: US Department of Commerce, National Institute of Standards and Technology, 2011.

10 Villarreal, Victor H., and David A. Crocker. "Better pavements through internal hydration." Concrete international 29.2 (2007), pp 32-36.

11 Ge, Zhi, Kejin Wang, and Zhili Gao. "Properties and early-age cracking potential of blended cement concrete." In Material Design, Construction, Maintenance, and Testing of Pavements: Selected Papers from the 2009 GeoHunan International Conference,( 2009), pp. $163-170$

12 Strategic Development Council "Roadmap 2030: The U.S. concrete industry technology roadmap. "Strategic Development Council, (2002).

13 Somesh Yendole, Prachi Rathod,Chinmay Mahaja, And Dipak Rathod "Review Paper On Retempring Unconcern And Their Effect, International Journal Of Research In Advent Technology,(2017), Pp 293-296.

14 Kiran M. Mane And Dr. D. K. Kulkarni,"Effect Of Retempring With Retarding Admixes On Property Of Concrete Subjected To Prolonged Mixing", Innovative Systems Design And Engineering, (2011) , Pp 26-33.

15 Bingöl, A. Ferhat, and İlhan Tohumcu. "Effects of different curing regimes on the compressive strength properties of self compacting concrete incorporating fly ash and silica fume." Materials \& Design 51 (2013), pp 12-18.

16 Osei, Daniel Yaw, Zakari Mustapha, and Mohammed DH Zebilila. "Compressive Strength of Concrete Using Different Curing Methods." Journal of Social and Development Sciences 10.3 (S) (2019), pp 30-38.

17 Kim, Kukjoo, Thanachart Subgranon, Mang Tia, and Michael Bergin. "Internally cured concrete for use in concrete pavement using accelerated pavement testing and finite-element analysis." Journal of Materials in Civil Engineering 28, no. 6 (2016): 04016011.

18 IS 10262:2009 Indian Standard Concrete mix proportioning guidelines.

19 IS 456: 2000 Plain and reinforced concrete code of practice.

20 IS 516: 1959 Method of tests for strength of concrete.

21 IRC: 44. (2017). Tentative guidelines for cement concrete mix design for pavements. New Delhi:Indian Road Congress.

22 IRC: 15. (2017). Code of practice for construction of jointed plain concrete pavements. New Delhi Indian Road Congress.

23 IRC 58. (2017). Guidelines for the design of plain jointed rigid pavements for highways. New Delhi: Indian Road Congress.

24 IS 456: 2000 Plain and reinforced concrete code of practice.

\section{AUTHORS PROFILE}

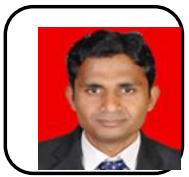

Vishal B Shinde, Research scholar, TEP, Civi Engineering Dept. at Sardar Vallabhbhai Patel National Institute of Technology, Surat, Gujarat, India, Email: shindevishal747@gmail.com

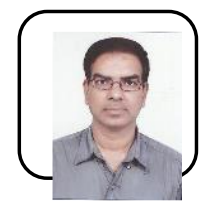

Dr.Rakesh Kumar, Professor, TEP, Civil Engineering Dept. at Sardar Vallabhbhai Patel National Institute of Technology, Surat, Gujarat, Email: krakesh1999@gmail.com

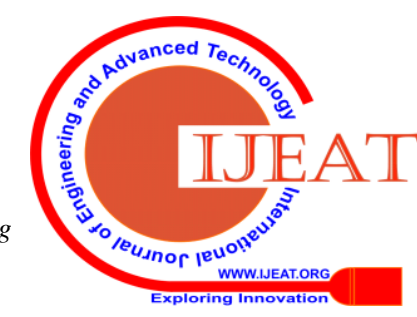

